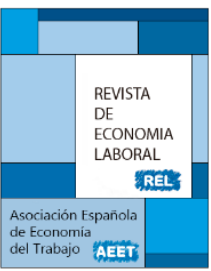

\title{
LA EFECTIVIDAD DE LAS REFORMAS LABORALES EN EL EMPLEO DE LOS JÓVENES
}

\author{
Inmaculada Cebrián y Gloria Moreno \\ Universidad de Alcalá \\ Recibido Julio 2015; Aceptado Octubre 2015
}

\section{Resumen}

La difícil situación de los jóvenes en el mercado de trabajo español ha dado lugar a que las reformas legislativas más recientes hayan incorporado al sistema nuevos instrumentos con el propósito de fomentar el empleo estable de este colectivo. El objetivo de este estudio es evaluar el impacto de estas medidas en el empleo de los jóvenes menores de 30 años desde el año 2007 y tratar de averiguar si se ha logrado, por un lado, mejorar los canales de acceso de la población joven al mercado de trabajo, y por otro lado, tratar de identificar si han aumentado los niveles de estabilidad laboral de estos trabajadores más jóvenes. Para ello se utiliza la información procedente de los registros de contratos del Servicio Público de Empleo Estatal (SEPE) y de la Muestra Continua de Vidas Laborales (MCVL). Los resultados de ambos análisis permiten concluir que los incentivos a la contratación indefinida en el caso de los jóvenes no han contribuido a mejorar la probabilidad de que un joven acceda a un empleo indefinido, ni tampoco han ayudado a mejorar el nivel de estabilidad que da el empleo indefinido a este colectivo.

Palabras clave: empleo juvenil, contratos indefinidos, bonificaciones e incentivos a la contratación, reducción cuotas Seguridad Social.

Clasificación JEL: J41 J63

\section{Abstract}

Young workers present a very difficult situation in the Spanish Labour Market. With the aim of improving it, the most recent labour market reforms have tried to promote the creation of new jobs as well as youth employment stability through reductions in social security contributions. This paper assess the impact of these new measures in the case of workers under 30 years old since 2007. The analysis tries to find out whether there has been, on the one hand, an improvement of how young people access to the labour market and, on the other hand, whether the levels of job security have increased for these younger workers. Data information comes from the records of Spanish Public Employment Service (SEPE) and the Continuous Sample of Working Lives (MCVL). The results from probabilistic and duration analysis support the hypothesis that the incentives for permanent contracts contributed to improve neither the likelihood that a young person access to a permanent contract, nor the level of stability of this type of employment, reductions in social security contributions.

Key words: youth employment, open-ended contracts, hiring subsidies and incentives JEL Classification: J41 J63

C Revista de Economía Laboral 


\section{Introducción}

Una de las consecuencias de la crisis económica que afecta a todos los países, tanto desarrollados como en vías de desarrollo, es el empeoramiento de la situación de los jóvenes en el mercado laboral. Cada vez es más difícil el acceso de los jóvenes al empleo, hasta tal punto que cada vez hay más jóvenes que renuncian a buscarlo y además, los que acceden al empleo se ven obligados a aceptar trabajos de baja calidad, renunciando a la estabilidad y a la seguridad laboral. Según la OIT 2013 la tasa de desempleo juvenil mundial alcanzó en este año el 12,6 por ciento, estando desempleados 73 millones de jóvenes. En el caso concreto de España, la tasa de ocupación de los menores de 30 años era del 35 por ciento en el último trimestre del 2014 según la Encuesta de Población Activa (EPA) y había un millón y medio de desempleados jóvenes que suponían el 27 por ciento del total de parados. Y las tasas de desempleo en ese trimestre alcanzaron el 67 por ciento en el caso de los menores de 20 años, el 49 por ciento para los que tenían entre 20 y 24 años y el 30 por ciento para aquellos entre los 25 y los 29 años. Estos datos sitúan a España como el país europeo con mayor tasa de desempleo juvenil. A esto hay que añadir que también los jóvenes españoles son los que padecen en mayor medida la temporalidad. Entre los pocos jóvenes que están empleados, la tasa de temporalidad supera el 50 por ciento. Esta temporalidad lleva aparejada una elevada rotación. En ocasiones, esta rotación es el resultado de la sucesión de contratos dentro de una misma empresa, la cual elude de esta manera la contratación indefinida. Según la información de la Muestra Continua de Vidas Laborales (MCVL) la población joven que ha tenido algún empleo entre 2005 y 2013 tiene por término medio 7,4 episodios de empleo de los cuales, 5,9 fueron temporales. Así mismo, según los datos de los registros del Servicio Público de Empleo (SEPE), durante el año 2014 aproximadamente un tercio de los contratos registrados correspondieron a trabajadores menores de 30 años, de los cuales, sólo el 6.9 por ciento fueron de carácter indefinido y el 43,1 por ciento con jornada a tiempo parcial ${ }^{1}$.

Uno de los problemas que genera esta situación de los jóvenes en el mercado laboral es la dificultad que tienen para emanciparse (Gregg Scutella y Wadsworth, 2004, Ayllón 2014) de tal manera que, según el Observatorio de Emancipación del Consejo de la Juventud de España,

\footnotetext{
1 En cambio, para el total de contratos registrados, la proporción de indefinidos fue ligeramente superior (8,1 por ciento), mientras que la de tiempo parcial fue menor $(35,1$ por ciento).
} 
en el tercer trimestre de 2014 solo el 21,8 por ciento de los menores de 30 años había logrado emanciparse, el resto vivía con sus padres.

Esta situación es una constante del mercado de trabajo español de las últimas décadas. Algunos trabajos apuntan que muchos jóvenes se encuentran atrapados en la temporalidad y que sólo algunos logran ocupar un empleo de carácter indefinido tras haber pasado cierto tiempo en la temporalidad (Güell y Petrongolo, 2007; Toharia y Cebrián, 2007; Cebrián y Toharia, 2008; García Pérez y Muñoz Bullón, 2011; García Pérez et al, 2014). La situación es similar en otros países, por ejemplo, en OIT 2013 se analiza para diez países desarrollados las transiciones de los jóvenes en el mercado de trabajo al final de la escolarización y también la transición al empleo estable. Los resultados del estudio muestran que ser hombre, tener mayores ingresos familiares, vivir en un entorno urbano son circunstancias que facilitan el acceso al empleo. Mientras que la transición a un empleo estable viene condicionada por las circunstancias específicas del mercado laboral en que se encuentren. Dadas las altas tasas de paro, los jóvenes tienden a aferrarse al trabajo que tienen, independientemente de su calidad. Este mismo informe señala en sus conclusiones que la mejora de los resultados en el empleo juvenil pasa por un conocimiento en profundidad de las cuestiones específicas de cada país en relación con el acceso al empleo y a las transiciones laborales de los jóvenes.

La consecución de la estabilidad ha sido un objetivo constante de los distintos gobiernos españoles, que han llevado a cabo sucesivas reformas para facilitar el acceso de los jóvenes al empleo estable. Entre estas medidas cabe destacar los cambios introducidos por el gobierno socialista en la reforma de 2011 y por el gobierno popular en la reforma de 2012 incentivando el empleo indefinido de los jóvenes menores de 30 años mediante bonificaciones a las cuotas empresariales a la seguridad social y deducciones fiscales. En la misma línea se encuentra la puesta en marcha por parte del Ministerio de Empleo y Seguridad Social de un conjunto de medidas contenidas en la "Estrategia de emprendimiento y empleo joven 2013-2016", que señala entre sus objetivos el aumentar la calidad y la estabilidad del empleo.

El esfuerzo presupuestario que requieren estas medidas es muy alto, por lo que resulta interesante poder analizar en qué medida las empresas utilizan estos modelos de contratación incentivada como vía de acceso al empleo para los jóvenes, así como hasta qué punto logran mejorar los indicadores de estabilidad de la población trabajadora más joven. En esta línea, el objetivo de este estudio es evaluar el impacto de estas medidas de contratación y tratar de averiguar si han logrado por un lado, mejorar los canales de acceso de la población joven al mercado 
de trabajo, y por otro lado, tratar de identificar si se han incrementado los niveles de estabilidad en el empleo de estos trabajadores más jóvenes. Para ello, tras unas reflexiones sobre el problema planteado, se analizan los contratos registrados por los jóvenes, diferenciando los distintos tipos de acceso y las características asociadas a éstos. A continuación, se estudia la estabilidad de los contratos indefinidos, teniendo en cuenta si se trata o no de contratos incentivados. Por último, se presentan las conclusiones de estos análisis.

\section{Planteamiento y datos}

La manera en la que la población joven accede al mercado de trabajo es importante, ya que puede condicionar la trayectoria laboral posterior de esta población. Algunos de estos efectos son explicados por la literatura que estudia de qué manera se pueden convertir en un estigma los momentos iniciales de la carrera laboral, lo que se conoce como "scarring effects" (The Economic Journal 2001, OCDE 2014). Estos trabajos analizan sobre todo de qué manera se ven proyectadas a largo plazo las consecuencias de las interrupciones debidas al desempleo. Estas ideas pueden aplicarse para analizar el efecto que puede tener sobre las trayectorias laborales el tipo de empleo y contrato con el que acceden al empleo los jóvenes. La evidencia empírica sugiere que la mayoría de los trabajadores que acceden al mercado laboral a través de un contrato temporal terminan teniendo peores resultados en términos de salarios y seguridad en el empleo. Incluso cuando la temporalidad es voluntaria las bajas tasas de transición a un contrato permanente generan desigualdades que tienden a persistir (OCDE, 2014).

En esta línea y como complemento a estos estudios, resulta interesante analizar de qué manera tiene lugar el acceso, si mediante un contrato temporal o indefinido, y hasta qué punto ayuda al trabajador joven a alcanzar un grado de estabilidad laboral que le permita ir acumulando experiencia. De hecho un objetivo de la Comisión Europea es, desde la base de la flexiseguridad, "la creación de puntos de apoyo eficaces que permitan a los trabajadores acceder al mercado laboral y progresar por medio de la movilidad ascendente que redundaría en beneficio de los ciudadanos y la sociedad" (Comisión Europea, 2007).

Junto con el acceso al empleo temporal o indefinido, hay que considerar la rotación como un factor más de estabilidad. La alta rotación que sufre la población joven española podría tener un lado 
"positivo", pues ayuda a que este colectivo pueda acceda al empleo al tiempo que se comparten los empleos existentes en la economía. Mientras haya cambios y se pase de unos empleos a otros, el individuo deja de estar desempleado. Ahora bien, en la medida en que esta rotación da lugar a que se tengan cortos periodos de empleo continuado, con pocas garantías de estabilidad y el individuo quede atrapado en este estado de inestabilidad, el avance dentro del mercado y los logros laborales quedan coartados. Un estudio de la Fundación Europea para la mejora de las condiciones de vida y trabajo (Eurofound) del año 2014 con datos de varios países de la Unión Europea, llega a la conclusión de que en Europa los trabajadores con un empleo temporal tienen una proporción de transiciones al desempleo mayores que los trabajadores con un empleo indefinido. Además, la crisis ha incrementado la proporción de trabajadores temporales que pasan a desempleados, en concreto, la proporción ha aumentado de un 11.6 entre 2006 y 2007 a un 18.5 por ciento entre 2009 y 2010 . Por lo que se puede decir que la crisis ha afectado a los trabajadores temporales jóvenes más por el incremento en la probabilidad de perder su trabajo que por la disminución de las oportunidades de encontrar un empleo estable. En este estudio también se pone de manifiesto la alta correlación negativa que existe entre las tasas de temporalidad de un país y la proporción de transiciones desde la temporalidad al empleo indefinido; en especial en los países mediterraneos como España, con elevadas tasas de temporalidad.

Hasta ahora siempre se ha pensado que el origen de esta elevada rotación estaba en la facilidad de los empresarios para recurrir a la contratación temporal, ya que un trabajador joven tiene más probabilidad de acceder al mercado a través de un empleo temporal, por resultarle más barato y flexible a la empresa. Esto explica que en los momentos de crisis, las empresas se ajusten a las nuevas condiciones de su demanda despidiendo o no renovando a los trabajadores temporales (Bentolila and Dolado, 1994). Este tipo de argumentos se suele utilizar a la hora de defender la conveniencia de reducir la protección del resto de los trabajadores, como principal alternativa para resolver el problema de la doble segmentación del mercado de trabajo español, que afecta de manera muy especial a los jóvenes. El segmento más perjudicado por los ajustes es aquel en el que se encuentran los trabajadores con contratos temporales, mientras que los que tienen contratos indefinidos pertenecen al segmento más favorecido porque están protegidos y supuestamente ven desde lejos cómo se producen los ajustes de plantilla de las empresas. Sin embargo, si miramos a España encontramos que la rotación afecta más a los trabajadores temporales, 
pero también hay trabajadores indefinidos que rotan, hasta tal punto, que hay estudios que ponen de manifiesto que al cabo de un año, casi el 40 por ciento de los contratos indefinidos han podido desaparecer (Cebrián, Moreno y Toharia, 2011).

Las diversas reformas laborales que han tenido lugar en España en los últimos años han tratado de incentivar el empleo estable a través de dos tipos de contratación indefinida: el contrato inicial que recibe algún tipo de bonificación en la cuota y el contrato indefinido por conversión de un temporal, que se ha mostrado, al contrario que el primero, como un medio especialmente valioso para mejorar las relaciones laborales y conseguir mayor grado de estabilidad (Cebrián, Moreno y Toharia 2011, Cebrián 2015). No obstante, a pesar de los esfuerzos de estas reformas, para algunos juristas, en el caso de los jóvenes la permanencia y resistencia a la temporalidad está relacionada con la propia legislación que así lo establece (Guamán, 2013). Por ejemplo, las reformas de 2012 y 2013 promocionan el empleo juvenil mediante la reducción de costes laborales, la flexibilización y reducción de los requisitos de formación y la creación de nuevas modalidades de contratación temporal sin causas para los jóvenes.

Otra cuestión a tener en cuenta es que desde la reforma de 2010 (Ley 35/2010) se encareció el uso de la contratación temporal (aumentando las cuotas empresariales y el coste de finalización de contrato) y desde 2012 (RDL 3/2012) se ha generalizado a todos los contratos indefinidos el coste de los despidos improcedentes (33 días por año trabajado con un máximo de 24 mensualidades) al que anteriormente sólo podían acceder los contratos indefinidos de fomento del empleo (Ley 12/2001). Además, ambas reformas modificaron las condiciones para que un despido fuese declarado objetivo, siendo especialmente flexibles las condiciones establecidas por la norma desde 2012. En este sentido resulta también interesante poder analizar si aumentar los costes de la contratación temporal al tiempo que se reducen los costes de protección de la contratación indefinida ha servido para mejorar el acceso al empleo indefinido y no han ampliado el flujo de salida desde este tipo de empleos empeorando el grado de estabilidad de la contratación indefinida. Por esta razón, podría resultar que al final lo que termina ocurriendo es que la tasa de rotación laboral aumenta en general como consecuencia del uso de contratos indefinidos menos protegidos ${ }^{2}$ (Cahuc, 2014). En cambio, la idea que subyace en los

\footnotetext{
2 Los modelos de protección del empleo, que establecen normas que restringen los despidos, tienen como principal objetivo mejorar el nivel de estabilidad de un empleo pero, sin embargo, condiciona los flujos de entrada y salida del empleo, lo que puede
} 
cambios introducidos por las reformas es que el contrato por tiempo indefinido es la principal fuente de seguridad y protección laboral como alternativa a la elevada temporalidad existen en el mercado de trabajo español.

Esta perspectiva se aplica al caso general español aunque también se utiliza como alternativa para la población más joven, ya que una gran parte de las medidas de incentivos a la contratación indefinida se han focalizado en el grupo de personas menores de 30 años. Entre otras medidas desde la publicación del RDL 3/2012 y la Ley 3/2012 hay que destacar, el "contrato de emprendedores" que potencia la contratación indefinida cuando la empresa contratante tiene menos de 50 trabajadores. Se introducen bonificaciones a las cotizaciones empresariales por contingencias comunes a la Seguridad Social, además de deducciones fiscales y ampliación del periodo de prueba hasta un año, lo que puede haber ayudado a la entrada de los jóvenes al empleo. El RDL 16/2013 posibilita que estos contratos se realicen a tiempo parcial.

También se introducen modificaciones de los contratos formativos y en prácticas (RDL 10/2011, Ley 3/2012 y Ley 11/2013). Hay que resaltar la implantación de la "Garantía Juvenil" (Hernández Diez y Gentile 2015) como un sistema global e integrado que permite a los jóvenes recibir una buena oferta de empleo, de formación continua, de prácticas o de formación de aprendices en un plazo de cuatro meses a partir del momento en que terminan sus estudios o se quedan en paro (Dictamen del Comité Económico y Social Europeo, "Promover el empleo juvenil"). Se establecen una serie de medidas de contratación y bonificación para incentivar el empleo de los menores de 25 años (RDL 8/2014). Por ejemplo, una nueva bonificación para la contratación indefinida de jóvenes beneficiarios de la Garantía Juvenil. Además se establece el contrato "primer empleo joven" (Ley 11/2013), en el marco de la "Estrategia de emprendimiento y empleo joven". Se trata de una modalidad del actual contrato eventual cuya causa se encuentra en la ausencia de experiencia del trabajador objeto del contrato (causa de carácter subjetivo) y se incentiva su transformación a indefinido. Con el objetivo de facilitar la inserción de algunos colectivos con dificultades en

terminar perjudicando la dinámica de flujos en el mercado de trabajo y por ende, los niveles de empleo. La hipótesis de partida es que las medidas de protección del empleo indefinido reducen el grado de rotación estos empleos, porque limitan tanto las entradas como las salidas, al tiempo que favorecen otros tipos de contrataciones con menos restricciones y menores costes de salida. Este puede ser el caso de España, donde la legislación sobre despidos establece condiciones diferentes para los trabajadores temporales y para los indefinidos. 
el mercado laboral, tales como los jóvenes sin experiencia laboral, se establecen los contratos indefinidos conocidos como de "tarifa plana" (RDL 3/2014, RDL 1/2015), que bonifican con una tarifa plana o un mínimo exento (desde 2015) las cotizaciones empresariales por contingencias comunes a la Seguridad Social.

En resumen, el marco legislativo ofrece un abanico de incentivos al empleo indefinido y resulta de interés conocer en qué medida se están utilizando para contratar a jóvenes y si los trabajadores que acceden directamente a un contrato indefinido tienen algunas características que los diferencien de los demás y si tienen mayor estabilidad en el empleo ${ }^{3}$.

La primera parte del estudio se realiza a partir de la información del registro de contratos del Servicio Público de Empleo Estatal (SEPE) ${ }^{4}$, describiendo el perfil de los trabajadores jóvenes que acceden a un empleo y de los puestos según los distintos tipos de contrato con los que acceden (indefinidos, temporales). El periodo de observación abarca desde el 1 de enero de 2007 hasta el 31 de diciembre de 2014. En primer lugar, se analiza la probabilidad de que una persona joven acceda al mercado de trabajo mediante un contrato temporal o un contrato indefinido, utilizando para ello un modelo logit. A continuación, dado que el objeto es estudiar el papel de los nuevos incentivos a la contratación indefinida, se estudia la probabilidad de acceder con un contrato indefinido de un tipo u otro, con un modelo multilogit. Ahora bien, los datos de contratos permiten estudiar sólo las entradas, no las salidas. Por ello, la segunda parte del análisis utiliza los datos de la Muestra Continua de Vidas Laborales $(\mathrm{MCVL})^{5}$, en su versión de panel entre 2007 y 2013, con el objeto de poder medir la duración de los empleos de los jóvenes y valorar en qué medida los incentivos a la contratación indefinida ayudan a lograr la tan ansiada estabilidad laboral ${ }^{6}$. En esta parte del análisis, el periodo de observación llega sólo

\footnotetext{
3 En el SEPE se puede encontrar un resumen del conjunto de incentivos a la contratación:

https://www.sepe.es/contenidos/que_es_el_sepe/publicaciones/pdf/pdf_empleo/bonificacio nes_reducciones.pdf

${ }^{4}$ Las autoras agradecen al SEPE la posibilidad de utilizar los microdatos del registro de contratos gracias al Convenio de colaboración firmado entre el SEPE y la Universidad de Alcalá.

${ }^{5}$ Las autoras agradecen a la Seguridad Social el haber podido disponer de estos datos.

${ }^{6}$ Los modelos estimados, tanto con datos de registros de contratos como con la MCVL se realizan únicamente para personas que tienen la nacionalidad española, debido a problemas detectados en los identificadores de los extranjeros.
} 
hasta el 31 de diciembre de $2013^{7}$. La utilización de la versión de panel de la MCVL garantiza que estén representados todos los episodios que se inscribieron a lo largo del periodo analizado.

Los resultados de ambos análisis permiten valorar en qué medida se está logrando mejorar el acceso y estabilidad del empleo juvenil, y si se están estableciendo las bases para reducir la segmentación laboral entre trabajadores indefinidos y temporales.

\section{El acceso de los jóvenes al empleo indefinido}

En este apartado se va a analizar qué ha sucedido con las entradas al empleo de los jóvenes desde el inicio de la crisis económica con el objetivo de detectar los posibles efectos de las medidas de fomento de la contratación indefinida que se han ido aplicando. Para ello se van a utilizar los contratos registrados en el SEPE, entre el primer trimestre de 2007 y el último de 2014, correspondientes a jóvenes menores de 30 años.

Durante este periodo, los contratos correspondientes a jóvenes supusieron el 40 por ciento del total de los contratos registrados, aunque la proporción fue disminuyendo desde un 47 a un 33 por ciento. Un efecto claro de la crisis fue la expulsión de muchos jóvenes del mercado de trabajo, a saber, si durante los años previos a la crisis los más jóvenes podían encontrar un empleo aún con poco nivel de cualificación, desde 2008 el panorama cambió y muchos de estos jóvenes que habían abandonado el sistema educativo para trabajar y ante el panorama del desempleo pasaron a ser inactivos (Rocha, 2012).

En el gráfico 1 puede verse que la tasa de actividad de los menores de 30 años pasó del 67 al 58 por ciento y que su tasa de ocupación disminuyó del 58 al 35 por ciento. Si bien la disminución fue mayor entre los de menos edad. Según la EPA, la tasa de actividad del grupo entre 16 y 19 años cayó del 30 por ciento en 2007 al 15 por ciento en 2014. También disminuyó entre los que tenían entre 20 y 24 años, pasando del 67 al 57 por ciento, mientras que la tasa de actividad de los que se encontraban entre los 25 y los 29 años se mantuvo más estable, en torno al 87 por ciento. El descenso de la tasa de ocupación se sucedió año tras año, salvo entre 2013 y 2014 que aumentó ligeramente (menos de un punto porcentual). La caída en la tasa de ocupación concuerda con la evolución de los contratos registrados de jóvenes y hay que señalar

\footnotetext{
${ }^{7}$ Este periodo es más reducido por no estar disponible la MCVL de 2014 en la fecha de elaboración del estudio.
} 
que durante todo el periodo la tasa de paro mostró una tendencia creciente, a pesar de la disminución de la población activa. Este resultado se debe a que, a pesar de que una parte de los jóvenes abandonan el mercado de trabajo o retrasan su incorporación, los que permanecen o acceden al mercado están en gran medida desempleados.

\section{Gráfico 1 Contratos registrados 2007-2014 menores y mayores de 30 años, proporción de menores sobre el total. Tasa de actividad y tasa de ocupación menores de 30 años}

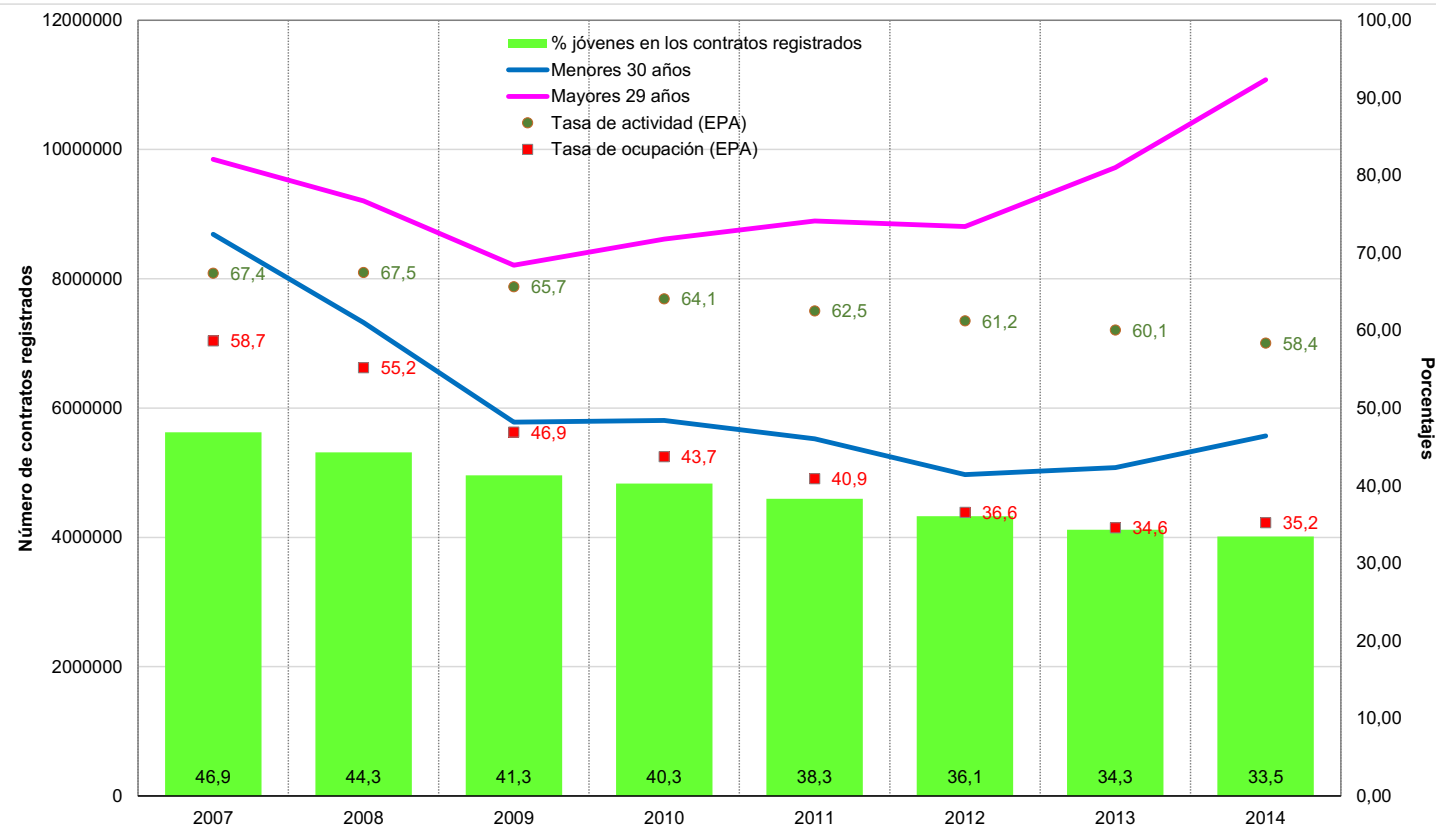

Fuente: EPA y microdatos del registro de contratos, SEPE.

Entre 2007 y 2014 se registraron más de 48 millones de contratos en los que el individuo tenía menos de 30 años, que corresponden a unos 10 millones de identificadores personales distintos ${ }^{8}$.

\footnotetext{
8 En los microdatos del registro de contratos no es posible identificar a todos los individuos, algunos casos aparecen sin la identificación completa. También se han detectado algunos problemas con los identificadores de los individuos de nacionalidad
} 
El acceso al empleo se produce mayoritariamente a través de contratos temporales, y esto es así para toda la población, no solo para los jóvenes. En el gráfico 2 se observa la distribución de los distintos tipos de contratos registrados para dos grupos de edad, menores de 30 años y mayores de 29 años, y esto para el periodo 2007-2014.

\section{Gráfico 2 Distribución de los contratos registrados 2007-2014, por tipo de contrato, menores de 30 y mayores de 29 años}

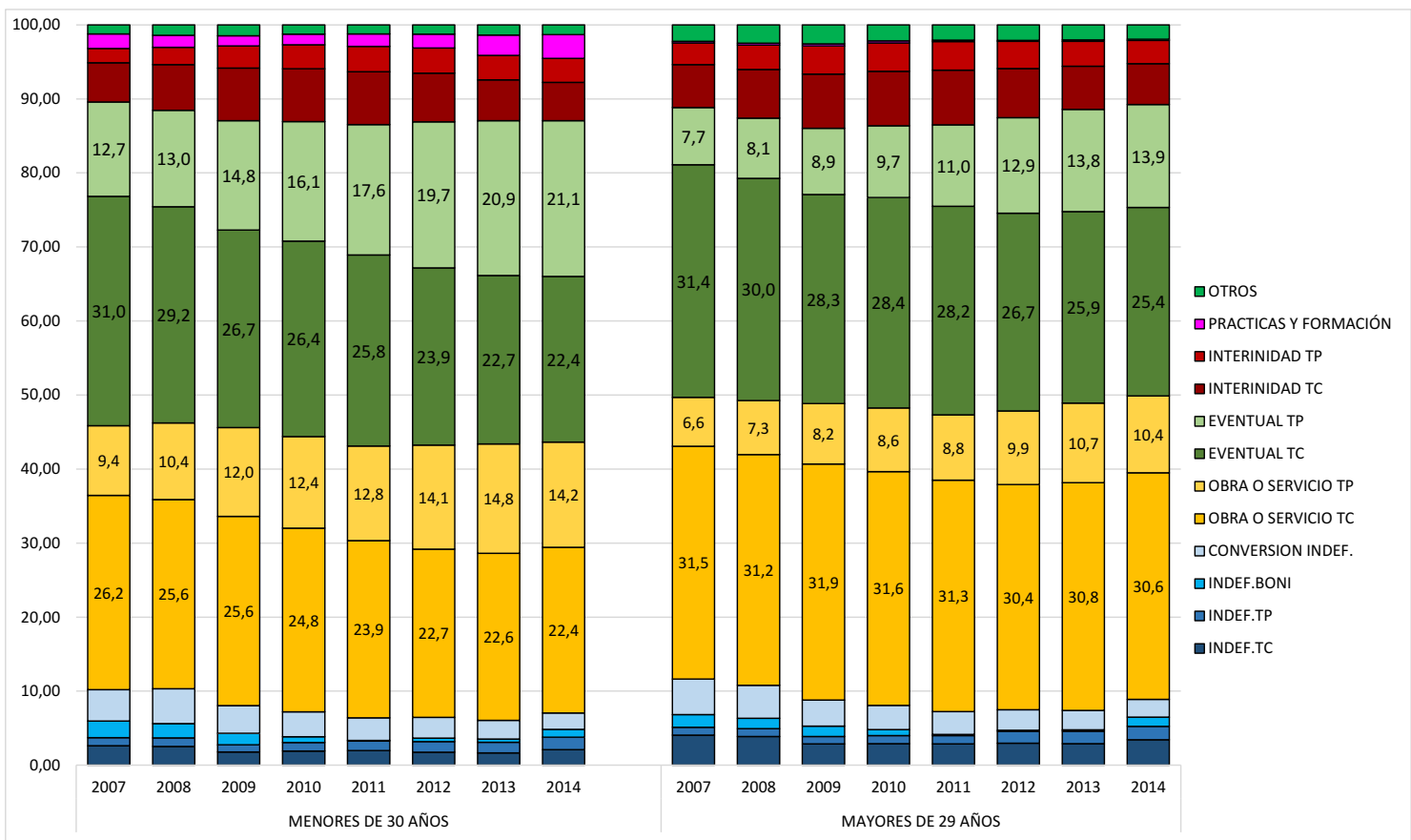

Fuente: microdatos del registro de contratos, $\mathrm{SEPE}^{9}$.

extranjera. Por lo tanto los datos referidos a individuos y no a contratos han de leerse con cautela.

${ }^{9}$ Las cifras de contratos registrados corresponden a los microdatos proporcionados por el SEPE. Estas cifras pueden no corresponder con los datos publicados por dos motivos, por un lado, con posterioridad a su publicación los registros se siguen actualizando. Y por otro lado,se han unido bajo la categoría de contratos indefinidos con bonificación todos aquellos contratos indefinidos iniciales que reciben algún tipo de incentivo, ya sea por bonificación o por deducción en la cuota empresarial a la Seguridad Social desde el año 2011. 
La contratación indefinida suponía en 2007 en torno al 10 por ciento de los contratos registrados por jóvenes y el 11,63 por ciento de los registrados por mayores de 29 años. En cambio, en 2014 esta proporción se redujo al 6,25 por ciento para los jóvenes y al 7,85 por ciento para el resto.

Los contratos con mayor peso son los temporales de obra o servicio y los eventuales, siendo estos últimos algo más comunes entre la población más joven. A lo largo del periodo se observa que ganan peso las modalidades a tiempo parcial frente a las de tiempo completo, en los dos grupos de edad. Si bien, los contratos temporales a tiempo parcial se dan en mayor proporción entre los jóvenes. Así, en 2007 los contratos temporales a tiempo parcial de los menores de 30 años son el 24,4 por ciento del total y en 2014 , el 38,8 por ciento, mientras que entre los mayores de 29 años las proporciones son del 17,2 y 27,7 por ciento respectivamente.

Los contratos en prácticas y de formación sólo suponen el 1,9 por ciento de los contratos registrados por jóvenes en 2007 aumentando ligeramente hasta llegar al 3,3 por ciento en 2014 .

El volumen total de contratos registrados ha caído un 10 por ciento entre 2007 y 2014, aunque en el caso de los menores la caída ha sido del 36 por ciento, mientras que entre los mayores de 29 años han aumentado un 10 por ciento. La evolución año a año se recoge en el gráfico 3, donde se resumen las tasas de variación anuales de los contratos según dos características: su tipología (indefinidos y temporales) y su jornada (tiempo completo y tiempo parcial) para menores de 30 y el resto. 
Gráfico 3 Tasas de variación anuales de los contratos registrados 2007-2014, por contratos indefinidos y temporales y tipo de jornada, menores de 30 años y mayores de 29 años

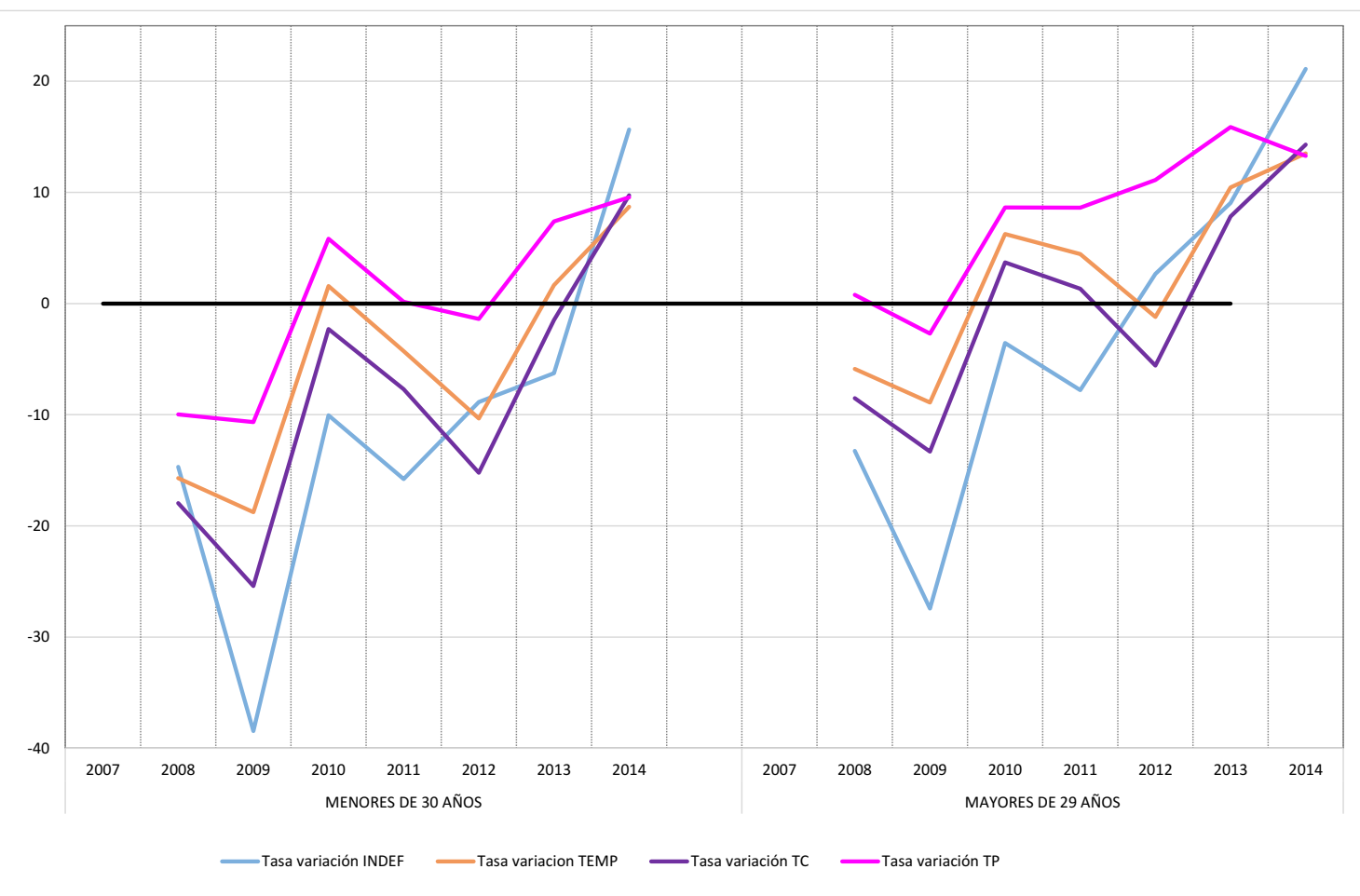

Fuente: microdatos del registro de contratos, SEPE.

En general, los contratos de jóvenes han caído más que los de mayores, así como la contratación indefinida y a tiempo completo se ha reducido más que la temporal y la de tiempo parcial. En el gráfico 3 además se observa que las caídas en el empleo indefinido registrado durante los años de crisis han sido mayores en el caso del colectivo más joven. Entre 2008 y 2009 se produce la mayor caída del empleo indefinido registrado, -38 por ciento y -27 por ciento, jóvenes y mayores respectivamente. La contratación indefinida creció en 2014 en ambos colectivos, aunque entre los más mayores empezó a crecer en el año anterior.

A pesar de que el fomento del empleo juvenil se ha tratado de articular a través de medidas que promueven el empleo estable no son muchos los contratos registrados por menores de 30 años que se hayan 
acogido a alguna bonificación o reducción en la cuota entre 2007 y 2014 . En el cuadro 1 puede verse la proporción de contratos incentivados.

Cuadro 1 Proporción de contratos de menores de 30 años con algún tipo de bonificación o reducción en la cuota, 2007-2014

\begin{tabular}{|c|c|c|c|c|c|c|c|c|c|}
\hline & 2007 & 2008 & 2009 & 2010 & 2011 & 2012 & 2013 & 2014 & Total $2007-14$ \\
\hline $\begin{array}{l}\text { Proporción de } \\
\text { contratos registrados } \\
\text { con bonificación }\end{array}$ & ,30 & ,22 & ,00 & ,29 & ,65 & ,64 &, 35 &, 25 & 2,34 \\
\hline $\begin{array}{l}\text { Proporción de } \\
\text { contratos registrados } \\
\text { con reducción en la } \\
\text { cuota }\end{array}$ & & & & & ,28 & ,38 & ,24 &, 54 & 1,16 \\
\hline
\end{tabular}

Fuente: microdatos del registro de contratos, SEPE.

En concreto, en todo el periodo analizado tan solo el 2,34 por ciento de los contratos de este colectivo tuvo alguna bonificación y a partir del 2011, el 1,16 por ciento alguna reducción en la cuota. También se observa que a partir de 2011, cuando se promueven las reducciones en cuota por primera vez con el RDL 1/2011, el uso de las bonificaciones disminuye.

Mención especial merecen algunos programas concretos de fomento del empleo que a pesar de lo esperado no han tenido apenas repercusión en los contratos registrados de los jóvenes. Por ejemplo, el contrato indefinido de apoyo a emprendedores no ha llegado ni al uno por ciento de los registrados. En 2012 solo se registraron 35.834, en 2013 fueron todavía menos 26.134 y en 2014 aumentan hasta 33.483. Por otro lado, los contratos de tarifa plana en el año 2014 de menores de 30 años fueron 47.835. Los contratos registrados al amparo del sistema nacional de Garantía Juvenil (RDL 8/2014) han sido únicamente 435, si bien es cierto que el sistema de Garantía Juvenil apenas comenzaba su andadura en el 2014 y habrá que analizar qué pasa durante los años venideros.

La disponibilidad de los microdatos del registro de contratos del SEPE permite conocer las características de las personas y de los puestos de trabajo a los que se accede. La distribución de estas características por tipos de contrato muestra algunas diferencias. Por ejemplo, los registros de mujeres suponen el 47 por ciento de todos los contratos registrados, aunque si se trata de contratos a tiempo parcial superan el 50 por ciento y entre los contratos de interinidad también, 
alcanzando cifras que están entre el 60 y el 80 por ciento, según el año. En el caso de los contratos indefinidos ordinarios hay más hombres, mientras que entre los bonificados hay más mujeres, lo cual no es de extrañar ya que una parte importante de estas bonificaciones se destinan a fomentar el empleo femenino.

Por lo que respecta a la edad media, los más jóvenes entran en mayor medida en contratos en prácticas o en formación y los más mayores en indefinidos a tiempo completo y también son más jóvenes los que entran en contratos a tiempo parcial que los de tiempo completo. La proporción de extranjeros es inferior al 30 por ciento en todos los tipos de contratos, siendo más alta en los contratos indefinidos a tiempo parcial o en los de obra o servicio a tiempo completo. El nivel de estudios muestra que la mayoría de los contratos corresponden a nivel secundario. Con un peso mayor de los estudios universitarios en los indefinidos, interinidades, prácticas y formación. El empleo registrado se concentra en el sector servicios en todas las modalidades contractuales y tan solo aparecen porcentajes destacados en la construcción y en la agricultura en los contratos temporales de obra o servicio. Las ocupaciones que presentan más peso en la contratación juvenil son aquellas que conllevan menor nivel de cualificación, destacando los contratos a tiempo parcial por el bajo nivel de estudios y con la excepción de los contratos indefinidos donde el nivel medio de estudios es más elevado. Los contratos indefinidos tienen más peso en las empresas pequeñas y son las Comunidades Autónomas de mayor nivel de renta per cápita las que registran mayor número de contratos de casi todas las categorías.

Tal y como se acaba de ver en el apartado anterior, los esfuerzos legisladores para potenciar el empleo en general y en concreto el empleo juvenil, se han dirigido a incentivar la contratación indefinida. Sin embargo, a pesar de estos incentivos, los contratos temporales son mayoritarios, independientemente de la edad del trabajador. Con el objetivo de conocer qué características son las que inciden en que una persona entre en un contrato indefinido o en uno temporal y sí las bonificaciones aumentan la probabilidad de entrar en un contrato indefinido, se ha estimado un modelo logit donde la variable dependiente es tener un contrato indefinido frente a uno temporal ${ }^{10}$.

10 La base de datos procedente de los registros de contratos del SEPE recoge información sobre la población joven que ha tenido algún contrato en el periodo. Por lo tanto, no es posible controlar por el sesgo de selección al no disponer de información sobre aquellos jóvenes que no estuvieron registrados. 
La estimación se realiza para el periodo 2007-2014, para jóvenes españoles menores de 30 años y se excluyen los contratos a discapacitados y fijos discontinuos, por sus peculiaridades en cuanto a bonificaciones. En el gráfico 4 se muestra el resultado de la estimación de la probabilidad relativa de que un contrato sea indefinido frente a que sea temporal, a lo largo de los trimestres considerados. El modelo controla por todas aquellas variables personales y del puesto de trabajo que de alguna manera pueden incidir en dicha probabilidad ${ }^{11}$. Las variables utilizadas como explicativas son: el sexo, la edad, el nivel de estudios, la comunidad autónoma del centro de trabajo, la rama de actividad, la ocupación, el tamaño de la empresa, el tipo de jornada, si el contrato tiene algún tipo de bonificación, la variación del empleo en el trimestre y el trimestre.

Gráfico 4 Probabilidad relativa de acceder a un contrato indefinido frente a un temporal 2007-2014, menores de 30 años, categoría de referencia primer trimestre de 2007

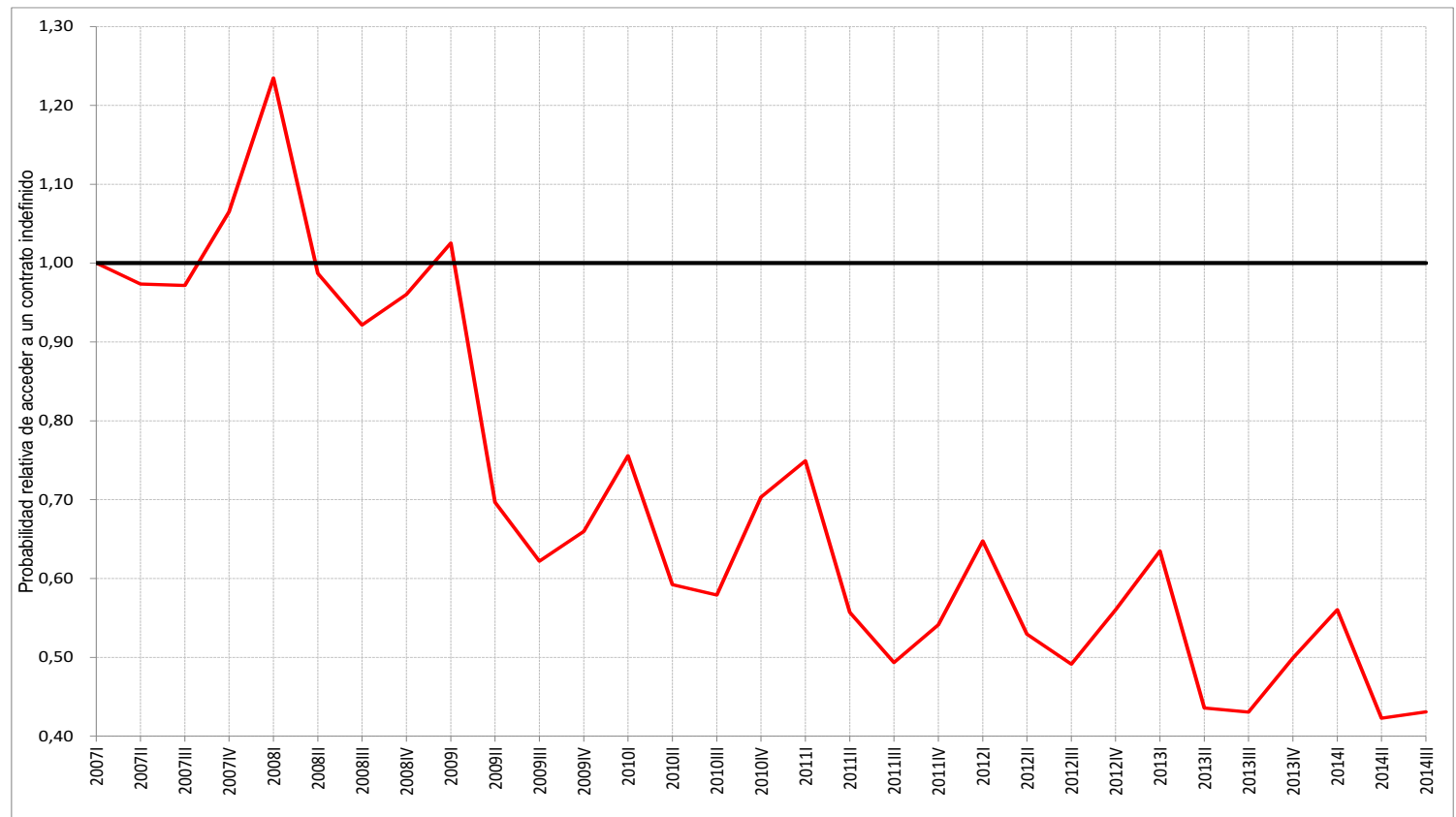

Fuente: microdatos del registro de contratos, SEPE.

\footnotetext{
${ }^{11}$ Los coeficientes de todas las variables y su significatividad se incluyen en el cuadro 1 del anexo.
} 
En el eje de ordenadas del gráfico 4 se recoge el valor de la probabilidad relativa de que se haya realizado un contrato indefinido frente a la probabilidad de que se haya realizado un contrato temporal. En el eje de abscisas aparecen reflejados los diferentes trimestres. Por tanto, cuando para un determinado trimestre la probabilidad relativa de que se haya realizado un contrato indefinido es mayor que la probabilidad de que se haya realizado un contrato temporal, el valor en el eje de ordenadas será mayor que 1 , mientras que si la probabilidad de que se haya realizado un contrato temporal es mayor que la de que se haya realizado un contrato indefinido el valor recogido en el eje de ordenadas será menor que 1 . Finalmente, si la probabilidad relativa de que se hayan realizado contratos indefinidos frente a temporales, en un determinado trimestre, no es significativamente distinta, el valor será igual a 1 (marcado por la línea que aparece en negro en el gráfico).

Los resultados de la estimación muestran la caída registrada en la contratación indefinida, tan solo en dos trimestres es mayor la probabilidad de registrar un contrato indefinido frente a un temporal, en los primeros trimestres de 2008 y 2009. El gráfico de probabilidad muestra la tendencia estacional del empleo más estable, se observa que en los primeros trimestres de cada año la probabilidad relativa de que el contrato registrado sea indefinido aumenta, a pesar de que en todo el periodo observado la probabilidad es decreciente.

Las probabilidades relativas respecto a las variables incluidas en el análisis corroboran lo que los valores medios de las distribuciones muestran. Es más probable tener un contrato indefinido para los hombres que para las mujeres y para los más mayores. El nivel de estudios también aumenta la probabilidad de tener un contrato indefinido, siendo la probabilidad relativa significativamente mayor en el caso de los estudios universitarios. Por ramas de actividad es en la industria extractiva y manufacturera, en el comercio y en las actividades financieras y de seguros donde la probabilidad de contrato indefinido frente a temporal es mayor. El grupo de ocupaciones con mayor probabilidad relativa de acceder con un contrato indefinido es el de administrativos, también las ocupaciones asociadas a mayor nivel de cualificación, como directivos y técnicos y profesionales de apoyo. Y la menor probabilidad de acceder a un indefinido se da en las ocupaciones de servicios de restauración, dependientes de comercio o trabajadores no cualificados. Por lo que respecta al tamaño del establecimiento, las empresas de menos de 4 trabajadores tienen una probabilidad relativa mayor de que los contratos registrados sean indefinidos, esta probabilidad disminuye según aumenta el tamaño del establecimiento. Madrid y Cataluña son las dos Comunidades Autónomas con mayor 
probabilidad relativa de contratos indefinidos y Andalucía la que presenta menor probabilidad relativa.

El resto de las variables explicativas muestran que la probabilidad de indefinido aumenta en los contratos a tiempo completo, en aquellos que tienen algún tipo de bonificación y en los trimestres en los que la variación del empleo es mayor, reflejando el efecto favorable del ciclo económico.

Con el fin de analizar qué factores inciden en la probabilidad de que una persona acceda al empleo indefinido con un contrato ordinario, bonificado o con una conversión se estima un modelo multinomial en el que la variable dependiente es el tipo de contrato indefinido. Las variables explicativas son las mismas que en la estimación anterior, salvo el que haya o no bonificación que en este caso se incluye en la variable independiente.

El gráfico 5 recoge los resultados de este análisis multinomial. En el eje de ordenadas se recoge el valor de la probabilidad relativa de que se haya realizado un contrato bonificado o una conversión, con o sin bonificación, frente a la probabilidad de que se haya realizado un contrato indefinido ordinario.

En el eje de abscisas aparecen reflejados los diferentes trimestres. Por tanto, cuando para un determinado trimestre la probabilidad relativa de que se haya realizado un contrato bonificado o una conversión, con o sin bonificación, es mayor que la probabilidad de que se haya realizado un contrato ordinario, el valor en el eje de ordenadas será mayor que 1 , mientras que si la probabilidad de que se hayan realizado contratos bonificados o conversiones es menor que la de que se hayan realizado contratos ordinarios el valor en recogido en el eje de ordenadas será menor que 1. Finalmente, si la probabilidad relativa de que se hayan realizado contratos bonificados o conversiones frente a los contratos ordinarios, en un determinado trimestre, no es significativamente distinta, el valor será igual a 1 (marcado por la línea negra).

El gráfico muestra que el único tipo de contratos que durante todo el periodo mantiene una probabilidad relativa mayor que los contratos indefinidos es el de conversiones no bonificadas, la línea que muestra la probabilidad relativa se mantiene por encima de la línea negra en todos los trimestres considerados. 


\section{Gráfico 5 Probabilidad relativa de acceder a un contrato indefinido ordinario frente a uno bonificado o una conversión bonificada o no, 2007-2014, menores de 30 años, referencia primer trimestre de 2007)}

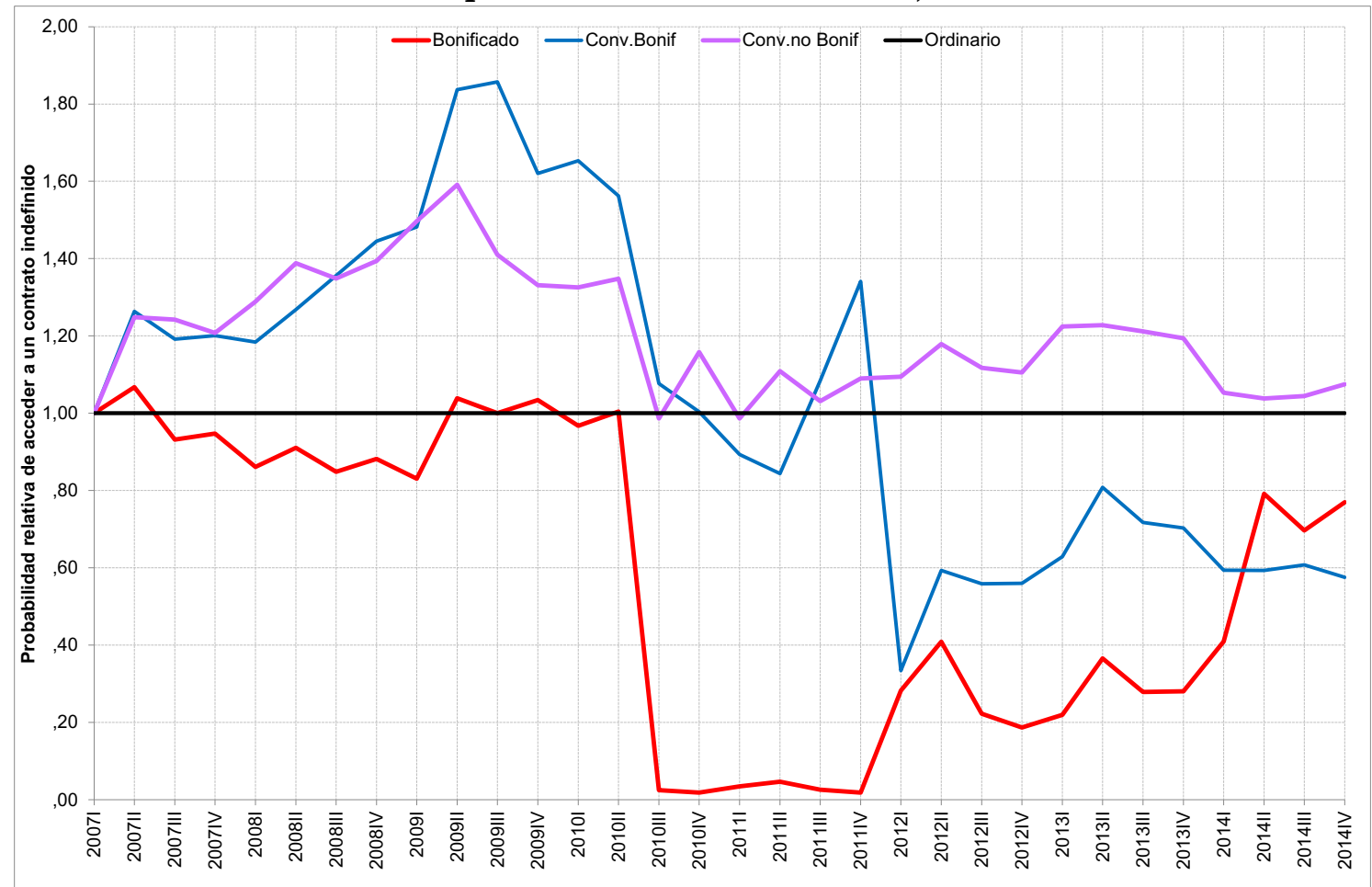

Fuente: microdatos del registro de contratos, SEPE.

Las conversiones bonificadas tienen mayor probabilidad relativa entre 2007 y el cuarto trimestre de 2010, luego la probabilidad cae por debajo de 1 durante los tres primeros trimestres de 2011, volviendo a aumentar en el cuarto trimestre de ese año, para nuevamente disminuir y mantenerse por debajo de la probabilidad relativa de indefinido ordinario hasta el último trimestre observado. En el caso de los indefinidos bonificados su probabilidad relativa está por debajo de la de los ordinarios, salvo entre el segundo trimestre de 2009 y el segundo de 2010, cuando la probabilidad es prácticamente la unidad. Sorprende la rápida caída a partir del segundo trimestre de 2010 hasta el último trimestre de 2011, momento a partir del cual muestra una recuperación moderada y continua coincidiendo con la aparición de las reducciones en la cuota empresarial a la Seguridad Social. 
En resumen, lo que se aprecia en los resultados de esta estimación es que la existencia de incentivos económicos no aumenta significativamente la probabilidad de que se acceda a un contrato indefinido bonificado, frente a uno ordinario, aunque muestre una tendencia creciente desde 2011. Mientras que la probabilidad de acceso a un indefinido a través de una conversión es mayor que a través de un contrato inicial ordinario, especialmente si son conversiones no bonificadas.

\section{La estabilidad laboral de los contratos indefinidos de los jóvenes: análisis de duración}

Tal y como se ha expuesto en el apartado 2, en el origen del debate sobre el papel de las instituciones y los costes de ajuste del factor trabajo se encuentra el problema de la elevada segmentación del mercado de trabajo español, problema que afecta principalmente a la población más joven. No cabe duda de que todos los factores determinantes de los flujos que se producen en el mercado laboral van a condicionar sobremanera a la población recién llegada, ya que lo primero y más importante para ella es poder llegar a tener un empleo. Por ello cabe preguntarse si las políticas activas puestas en marcha recientemente por el gobierno español, que requieren de un importante esfuerzo presupuestario, son las más adecuadas para mejorar la empleabilidad y estabilidad de los jóvenes. El análisis de estabilidad de los contratos indefinidos que se realiza a continuación trata de aportar algo de luz a esta cuestión.

El estudio se realiza a partir de los datos de la Muestra Continua de Vidas Laborales (MCVL) correspondientes a los episodios de empleo indefinido iniciados entre enero de 2007 y diciembre de 2013 por personas menores de 30 años. La principal ventaja de esta base de datos es que permite conocer el momento de inicio y final, si es que tiene lugar, de los diferentes tipos de contratos indefinidos. Al igual que en el registro de contratos del SEPE se puede identificar si son contratos iniciales o por conversión de contratos temporales, así como si se acogen a alguna bonificación o reducción de las cuotas que paga el empresario a la Seguridad Social. Además, también se puede identificar si se trata de un contrato de jornada a tiempo parcial o completo, definidos según la clasificación de contratos ${ }^{12}$.

\footnotetext{
${ }^{12} \mathrm{Al}$ igual que ocurría con la base de datos procedente de los registros de contratos del
} SEPE, no es posible controlar por el sesgo de selección al no disponer de información 
La muestra está inicialmente formada por 78.712 episodios de empleo indefinido, correspondientes 75.227 jóvenes menores de 30 años, que se iniciaron entre el 1 de enero de 2007 y el 31 de diciembre de 2013. El Cuadro 1 recoge dos tipos de información sobre el periodo de vida de estos episodios, que han sido clasificados según la tipología de contrato a la que corresponden, incluida la información sobre la jornada, y el año en el que se inició el episodio. La primera parte del cuadro se refiere al porcentaje de casos en los que el episodio de empleo indefinido seguía en vigor a finales del año 2013 y la segunda parte presenta los valores medios de la duración relativa del episodio, medida en porcentaje. Esta medida de la duración permite conocer qué parte del tiempo potencial estuvo vigente el contrato.

Cuadro 2 Porcentaje de contratos indefinidos iniciados entre 2007 y 2013 "vivos" el 31 de diciembre de 2013, y su duración media relativa, según el año de inicio, el tipo de contrato y la jornada laboral, población menor de 30 años

\begin{tabular}{|c|c|c|c|c|c|c|c|c|c|}
\hline & Año de inicio & 2007 & 2008 & 2009 & 2010 & 2011 & 2012 & 2013 & Total \\
\hline \multicolumn{10}{|c|}{ Contratos "vivos" el 31 diciembre 2013 (\%) } \\
\hline \multirow{4}{*}{ 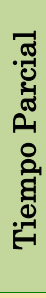 } & Ordinarios &, 5 & ,0 & 4,2 & 8,7 & 4,9 & 8,6 & 4,1 & 7,1 \\
\hline & Iniciales bonificados & ,9 &, 5 & 2,6 & 4,7 & 0,8 & 7,3 & 9,0 & ,8 \\
\hline & $\begin{array}{l}\text { Conversiones } \\
\text { bonificadas }\end{array}$ & 6,4 & 0,0 & 5,8 & 6,5 & 8,8 & 8,9 & 5,0 & 2,0 \\
\hline & $\begin{array}{l}\text { Conversiones no } \\
\text { bonificadas }\end{array}$ & 3,3 & 1,8 & 4,3 & 1,0 & 1,7 & 6,6 & 0,7 & 0,9 \\
\hline \multirow{4}{*}{ 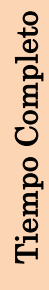 } & Ordinarios & 1,8 & 6,1 & 0,8 & 8,8 & 7,1 & 9,0 & 9,5 & 5,6 \\
\hline & Iniciales bonificados & ,8 & 3,5 & 9,0 & 2,8 & 4,2 & 7,9 & 0,0 & 3,2 \\
\hline & $\begin{array}{l}\text { Conversiones } \\
\text { bonificadas }\end{array}$ & 6,9 & 4,9 & 2,7 & 1,4 & 1,0 & 0,3 & 3,6 & 8,0 \\
\hline & $\begin{array}{l}\text { Conversiones no } \\
\text { bonificadas }\end{array}$ & 7,9 & 4,2 & 1,8 & 1,8 & 8,5 & 5,8 & 6,8 & 3,4 \\
\hline
\end{tabular}

sobre la población joven que no ha tenido ningún contacto con la Seguridad Social en el periodo analizado. 


\begin{tabular}{|c|c|c|c|c|c|c|c|c|c|}
\hline & \multicolumn{9}{|c|}{ Duración relativa media (\%) } \\
\hline \multirow{4}{*}{ 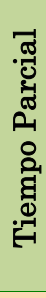 } & Ordinarios & 8,8 & 4,2 & 4,2 & 8,0 & 4,9 & 8,1 & 6,8 & 4,0 \\
\hline & Iniciales bonificados & 6,5 & 1,6 & 1,8 & 2,7 & 1,6 & 0,9 & 4,2 & 4,5 \\
\hline & $\begin{array}{l}\text { Conversiones } \\
\text { bonificadas }\end{array}$ & 6,9 & 5,3 & 4,3 & 7,8 & 4,7 & 8,7 & 9,1 & 1,4 \\
\hline & $\begin{array}{l}\text { Conversiones no } \\
\text { bonificadas }\end{array}$ & 5,5 & 4,1 & 8,8 & 5,9 & 5,0 & 5,7 & 0,3 & 1,3 \\
\hline 융 & Ordinarios & 8,5 & 4,7 & 1,1 & 8,9 & 6,8 & 6,4 & 0,3 & 3,0 \\
\hline 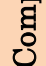 & Iniciales bonificados & 6,8 & 1,6 & 0,2 & 5,6 & 0,5 & 5,4 & 7,0 & 1,0 \\
\hline ․ㅠㅁ & $\begin{array}{l}\text { Conversiones } \\
\text { bonificadas }\end{array}$ & 2,2 & 8,0 & 6,2 & 1,2 & 8,9 & 6,9 & 7,7 & 7,8 \\
\hline$\stackrel{D}{\circledR}$ & $\begin{array}{l}\text { Conversiones no } \\
\text { bonificadas }\end{array}$ & 0,8 & 7,6 & 6,5 & 4,3 & 0,0 & 1,0 & 3,5 & 3,2 \\
\hline
\end{tabular}

Fuente: MCVL, 2007-2013.

Los datos del cuadro 2 ponen de manifiesto que los contratos indefinidos por conversiones de un contrato temporal tienen un porcentaje mayor de contratos "vivos" al finalizar el periodo y, dentro de este colectivo, son más estables los que se acogen a alguna bonificación. Entre los contratos iniciales, los llamados ordinarios, que son los contratos iniciales que no están bonificados, son más estables que los que reciben alguna bonificación. Este mismo resultado se obtiene al analizar los valores de las duraciones medias relativas porque en general, la duración media relativa bruta es más baja en los empleos a tiempo parcial y en los contratos iniciales bonificados. Las conversiones son más estables y las jornadas a tiempo completo también.

Ahora bien, este tipo de análisis adolece de ciertas deficiencias en tanto en cuanto no tiene en cuenta ni el momento en el que tuvo lugar el inicio de la relación, ni las características de los agentes implicados. Por ello, se utiliza un modelo de duración de Cox para estudiar qué determinantes explican la duración de los empleos que se iniciaron mediante un contrato indefinido en el periodo de observación ${ }^{13}$.

La variable dependiente es la duración del contrato. Los contratos que siguen vigentes al finalizar el periodo de observación se

\footnotetext{
${ }^{13}$ Una explicación detallada sobre este tipo de modelos, su metodología y conveniencia para este tipo de análisis puede encontrarse en Cebrián y Moreno (2011).
} 
considera que están "vivos" y por eso están censurados, ya que el individuo se encuentra en el sistema de la Seguridad Social con el mismo contrato en la misma empresa y no se puede conocer cuánto tiempo más durara esa relación laboral. En cambio, si el contrato ya no existe, porque consta que se ha dado de baja en el sistema de la Seguridad Social, se considera que ha "fallecido", siguiendo la terminología de los modelos de supervivencia.

Los determinantes de esta duración pueden tener que ver tanto con las características propias de la persona que firma el contrato como con las del puesto de trabajo que va a ocupar. Por ello, las variables explicativas que se van a utilizar en los modelos son similares a las utilizadas en los modelos de probabilidad realizados a partir de los registros de contratos. En concreto, las variables analizadas corresponden a las características personales, como el sexo y la edad, además de la antigüedad en el sistema de la seguridad social que se utiliza como un indicador de experiencia laboral. También se incorporan las características del puesto de trabajo referidas a la comunidad en la que está inscrita la empresa, la rama de actividad, el tipo de empresa y el tamaño del establecimiento; el grupo de cotización, que sirve como proxy del nivel de ocupación, el tipo de contrato, y la jornada laboral si es a tiempo completo o parcial. Y algunas variables que tratan de recoger el ciclo económico como son el trimestre en el que se inicia el episodio de empleo indefinido analizado y la tasa de variación del empleo en la Comunidad Autónoma correspondiente.

En el Cuadro 2 del Anexo se recogen las tasas de riesgo y el grado de significatividad de cada variable explicativa incorporada en el análisis. Los valores de la tasa de riesgo, también conocida como tasa de salida, permiten conocer cuál es la probabilidad de que el suceso analizado, en este caso el empleo en un determinado puesto de trabajo, deje de existir con el paso del tiempo. Los resultados del modelo dejan entrever algunas cuestiones interesantes.

En primer lugar, cabe destacar que no se observan diferencias significativas entre la duración de los empleos de los hombres y mujeres menores de 30 años ${ }^{14}$. Así como que la edad juega a favor de que la duración del empleo crezca, de modo que el individuo va ganando estabilidad laboral a lo largo de su vida. Luego los trabajadores de más edad, aunque sean jóvenes, tienen mayor probabilidad de entrar en un

\footnotetext{
14 Una posible explicación es que en esa etapa vital todavía no han llegado a tener cargas familiares que puedan estar condicionando sus periodos de empleo. Sin embargo, con los datos utilizados no es posible contrastarlo.
} 
contrato indefinido y además cuando lo hacen, es más probable que dure más tiempo.

Hay muy pocas Comunidades Autónomas en las que los resultados obtenidos difieran significativamente del caso de Andalucía, que es la Comunidad de referencia. Ahora bien, resulta sorprendente que en la Comunidad de Castilla la Mancha, la tasa de riesgo sea menor que 1 y que, en cambio, en Cataluña o Madrid la tasa de riesgo sea mayor que 1 , ya que esto indica que en dos de las Comunidades con mayor renta, es más probable que el empleo dure menos tiempo que en Andalucía. A pesar de ser estas dos Comunidades en las que es más probable que un joven entre en un contrato indefinido. En cambio, en Castilla la Mancha, los contratos indefinidos duran más.

En relación con el sector de actividad, sólo en las ramas de energía e intermediarios financieros la tasa de riesgo es menor que en la industria manufacturera. En este sector, junto con el comercio y las actividades financieras, la probabilidad de entrada es mayor.

El grupo de ocupación no se muestra como una variable significativa, siendo significativas sólo dos categorías: jefes administrativos $\mathrm{y}$ ayudantes no titulados, en ambos casos con duraciones más largas. Y de nuevo se trata de ocupaciones con las que es más probable que se acceda con un contrato indefinido.

A pesar de la incidencia negativa de la crisis económica, la incorporación al empleo a lo largo de los años más duros no parece afectar negativamente a la duración del empleo, ya que todos los trimestres presentan a partir del tercero de 2008 valores significativos y tasas de riesgo menores que uno.

El tipo de empresa en la que se inscribe la relación laboral es importante sólo si se trata de sociedades diferentes a las sociedades anónimas, en cuyo caso la probabilidad de que dure el empleo es menor, y si se trata del sector público, en cuyo caso, esta probabilidad es mayor.

Para terminar, hay que destacar los resultados en relación con el tipo de contrato, información que se representa en los perfiles de duración dibujados en el gráfico 6. Estos perfiles dibujan la tasa de supervivencia en función del tipo de contrato. Como se puede ver también en los valores de significatividad del Cuadro 2, no hay diferencia entre los contratos de iniciales a tiempo completo. En cambio, sí hay diferencia entre los contratos iniciales a tiempo completo y los que son a tiempo parcial, mostrando estos últimos unos perfiles de duración más bajos, por lo que se puede afirmar que duran menos. En cambio cualquier contrato por conversión de un temporal dura más, sobre todo si está acogido a algún tipo de incentivo que reduzca la cuota empresarial a la Seguridad Social. Un detalle interesante del análisis 
que hay que mencionar es que las diferencias en duración entre las conversiones y los contratos iniciales son importantes. El análisis realizado en el apartado anterior muestra que el acceso a un contrato indefinido vía una conversión de un contrato temporal (con o sin bonificación), es más probable que a través de un contrato inicial, independientemente de que tenga o no bonificación.

Obsérvese que al finalizar el que sería el primer año de vida de un contrato inicial, quedan "vivos" menos del 60 por ciento de los iniciados, cayendo incluso por debajo del 50 por ciento los iniciales bonificados. En cambio, la tasa de supervivencia de las conversiones puede llegar a ser hasta 20 puntos porcentuales mayor tras el primer año y alcanzar esas tasas en el segundo año. Ahora bien, no cabe duda de que es costoso para la sociedad que los contratos indefinidos, en los que se cree como los más estables y seguros, desaparezcan a tal ritmo que transcurridos 5 años no queden vivos ni el 40 por ciento, y en algunos casos, ni siquiera el 20 por ciento. Evidentemente estos resultados cuestionan la durabilidad, estabilidad y seguridad del empleo indefinido en el mercado de trabajo español.

Además, es igualmente importante destacar que al observar el resultado de la supervivencia por tamaño del establecimiento, resultados recogidos en el gráfico 7 , se aprecia que efectivamente, las empresas más grandes son las que garantizan que el empleo sea de más larga duración, mientras que las más pequeñas son las que tienen una probabilidad mayor de que el empleo termine. Esto viene a demostrar que hay una relación positiva entre la estabilidad y el tamaño del establecimiento. Sin embargo, como se vio en el apartado anterior, la probabilidad de acceso al empleo indefinido guarda una relación negativa con el tamaño de la empresa a pesar del peso que tienen las pequeñas y medianas empresas en el empleo en general y en el juvenil en particular. Por ejemplo, con los datos de contratos registrados se observa que en el año 2014 el 63 por ciento de los contratos registrados por menores de 30 años tuvo lugar en establecimientos con menos de 50 trabajadores. 


\section{Gráfico 6 Perfiles de supervivencia según el tipo de contrato}

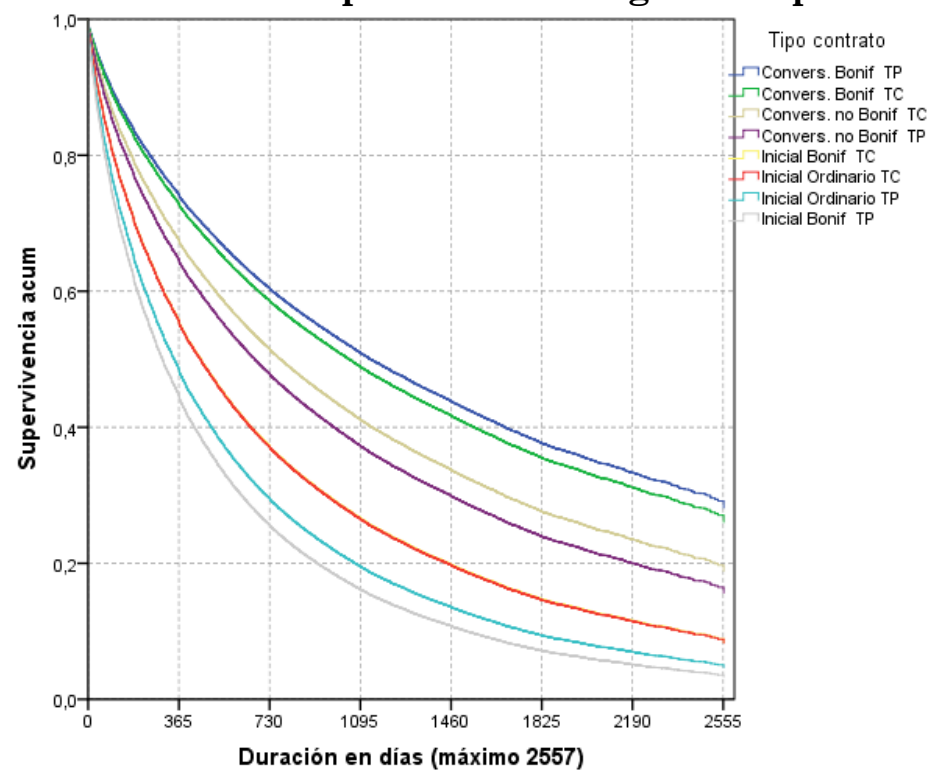

Fuente: MCVL, 2007-2013.

Gráfico 7 Perfiles de supervivencia según el tamaño del establecimiento

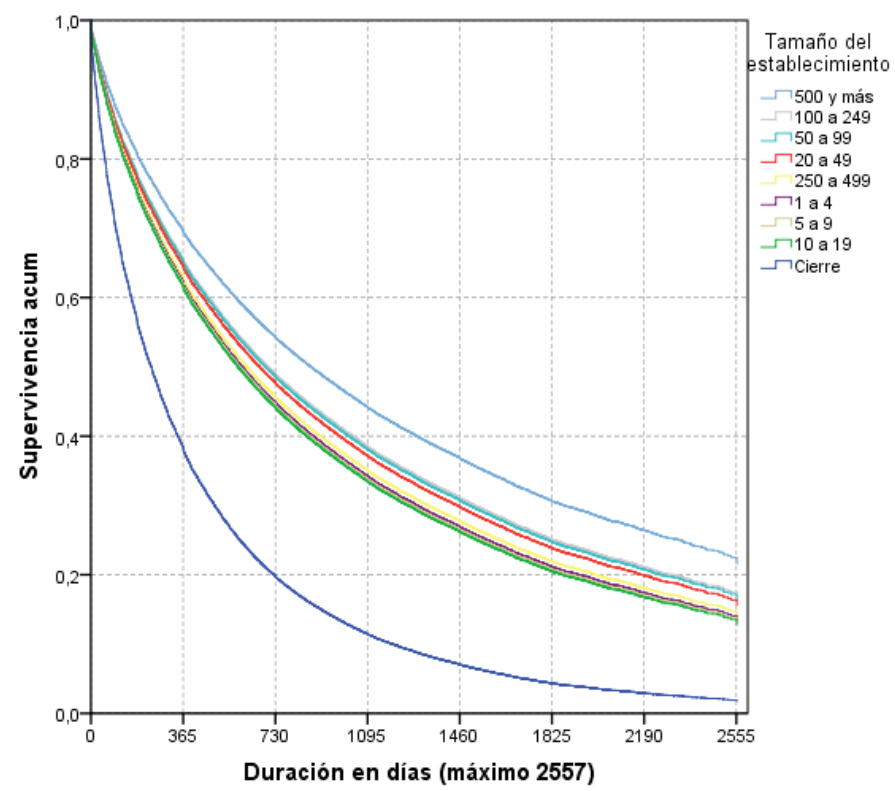

Fuente: MCVL, 2007-2013. 


\section{Conclusiones y comentarios finales}

La crisis económica ha supuesto en España un aumento de la tasa de paro hasta alcanzar uno de los niveles más elevados del conjunto de países de la Unión Europea y los jóvenes han visto agravada su situación en el mercado de trabajo más que ningún otro colectivo, con más de un millón y medio de desempleados a finales de 2014. A esto hay que unir el hecho de que el acceso al empleo se produce principalmente a través de contratos temporales, con altas tasas de rotación y escasa estabilidad. La evidencia empírica demuestra que el acceso a estos empleos no consigue la plena inserción laboral y alternan estos empleos con periodos de desempleo, lo que coarta su proyecto vital y retrasa su emancipación.

El fomento del empleo juvenil es un objetivo presente en los programas de todos los gobiernos, por ello, a lo largo de las últimas décadas se han ido estableciendo medidas que abarcan desde proyectos a largo plazo, como las reformas del sistema educativo con el fin de mejorar la empleabilidad de los jóvenes, hasta medidas que pretenden tener un impacto inmediato en el empleo como son los incentivos a la contratación indefinida.

El paradigma de la estabilidad es el empleo, y en particular, el empleo con un contrato de carácter indefinido. Por este motivo, este trabajo analiza la entrada en el empleo de los jóvenes a través de contratos de carácter indefinido, identificando en qué medida los incentivos a la contratación indefinida juegan un papel relevante, para estudiar posteriormente cuánto duran estos empleos y de qué depende su duración.

Tras los diversos análisis realizados, no parece que pueda concluirse que las bonificaciones o las reducciones en las cuotas a la cotización de la Seguridad Social, que pretenden equiparar los costes de los diferentes tipos de contratación, favorezcan la incorporación de los jóvenes al empleo indefinido. Del estudio de los datos de los registros de contratos del SEPE se desprende que la contratación indefinida representa una proporción muy pequeña de todos los contratos registrados, y no se observa que la existencia de incentivos a la contratación indefinida haya logrado aumentar la probabilidad de que un joven entre en un empleo con un contrato indefinido. De hecho, la probabilidad cae a lo largo de todos los trimestres analizados en el periodo comprendido entre 2007 y 2014.

Además, el hecho de que sea muy baja la probabilidad de que se utilicen los incentivos de la contratación indefinida y sólo sean las 
conversiones las que logren tener una probabilidad mayor que el contrato inicial ordinario pone de manifiesto al menos dos cuestiones interesantes. En primer lugar, estos resultados apuntan hacia la idea de que la decisión de contratar o no a un trabajador con un contrato indefinido tiene más que ver con las necesidades productivas de la empresa que con la facilidad para hacerlo. Si la empresa no necesita tener un trabajador que pueda establecer lazos de fidelidad con ella, o que requiera de cierta formación y estabilidad en la empresa, preferirá utilizar los resquicios que le permita la ley para contratar por la vía de la temporalidad. Y en segundo lugar, una vez más se observa que las medidas de fomento tienen un importante efecto "peso muerto", deduciéndose que los pocos contratos indefinidos que se realizan en España se habrían realizado en cualquier caso, con independencia de que se reduzcan los costes de las contrataciones vía incentivos. Además, en algunos trimestres del periodo analizado, la legislación vigente hizo extensiva la posibilidad de incentivar los contratos dirigidos a prácticamente todos los colectivos quedando diluido el efecto beneficioso que pudieran tener sobre colectivos específicos, por ejemplo, como es el caso de los jóvenes.

No cabe duda de que el escaso papel que tiene la contratación indefinida tiene mucho que ver con es el fenómeno conocido como la "cultura de la temporalidad" que impera entre el empresariado español y con el escaso control de las autoridades competentes ante las posibles situaciones de abuso de la causalidad de la contratación temporal (Toharia 2005).

Una explicación posible de por qué las empresas se ven poco atraídas por las modalidades de contratación incentivada puede ser que todas ellas conllevan la restricción de que la empresa debe mantener el tamaño neto de la plantilla durante al menos 3 años, o el tiempo que dure la bonificación. Esta rigidez puede suponer un mayor coste para la empresa que el hecho de tener que correr con los costes laborales de entrada y salida de un contrato indefinido sin bonificar.

En relación con la estabilidad y seguridad que supuestamente proporciona el empleo indefinido, el estudio de la duración de la contratación indefinida y las diferencias observadas entre los tipos de contratos, iniciales o por conversión, con incentivos o sin ellos, pone de manifiesto que los jóvenes padecen de un elevado grado de inseguridad, ya que pasado el primer año de contrato, la probabilidad de haber finalizado la relación es por término medio del 45 por ciento. Aunque hay diferencias significativas entre los contratos indefinidos iniciales y las conversiones, ya que estás se muestran más estables y sólo el 30 por ciento desaparece a lo largo de su primer año de vida. Este resultado 
pone nuevamente de manifiesto el hecho de que las empresas españolas aprovechan la contratación temporal para realizar una "cata" del trabajador con el fin de averiguar su nivel de competencia y adecuación a las necesidades de su empresa. De modo que una vez hecha la comprobación, la empresa accede a ofrecerle un contrato indefinido una vez pasado el filtro de la temporalidad. Este hecho pone de manifiesto que el periodo de prueba que tienen los contratos indefinidos parece ser insuficiente para las empresas. Quizás, por esa razón, el gobierno actual decidió incorporar a la legislación laboral el contrato de emprendedores que conlleva un año de prueba. Ahora bien, tampoco parece suficiente para las empresas poder tener disponible a un trabajador durante 365 días sin coste de despido alguno y, en muchos casos, además, con la posibilidad de disfrutar de incentivos, ya que como se ha demostrado, el registro de contratos indica que son muy pocos los contratos que se han firmado por esa vía.

En relación con las características analizadas, no hay diferencias significativas entre los hombres y las mujeres menores de 30 años. Sin embargo, hay algunas variables explicativas de la contratación indefinida y de la duración de estos contratos a las que se debe prestar atención.

Hay algunas ramas de actividad como la industria manufacturera o el sector energético que favorecen la entrada en el empleo indefinido y su duración. Esto mismo ocurre con las ocupaciones más cualificadas. Sin embargo, hay otras características en las que los efectos analizados van en sentido contrario. Este es por ejemplo, el tamaño de la empresa. Volviendo sobre el contrato de emprendedores, recuérdese que va dirigido a empresas de menos de 50 trabajadores. De acuerdo con los resultados de los modelos de probabilidad de acceder a un contrato indefinido, ejerce un efecto positivo que la empresa sea pequeña; en cambio, en los modelos de duración, las empresas más pequeñas tiene duraciones más cortas. Por lo tanto, hay un efecto positivo porque mejora la entrada pero podría decirse que por poco tiempo. Luego, el saldo neto final puede ser cero en el corto-medio plazo.

Algo parecido ocurre con las Comunidades Autónomas. De acuerdo con los modelos de acceso, aquellas Comunidades con mayor renta, como son Madrid y Cataluña, ejercen un efecto positivo sobre la entrada en la contratación indefinida. En cambio, cuando se estudia la duración de estos contratos, se observa que la tasa de riesgo de que termine el contrato es mayor. Por lo tanto, de nuevo, podría ocurrir que el saldo neto final, en términos de empleo, sea en el corto-medio plazo cero. 
También hay que destacar el hecho de que la probabilidad de entrada en un contrato indefinido sea mayor con una jornada a tiempo completo que a tiempo parcial, así como también es más probable que el contrato dure más.

En suma, si la contratación indefinida y su fomento no está dando lugar a los resultados esperados, y los contratos temporales siguen ocupando un lugar prioritario, cabe preguntarse hasta qué punto las políticas del mercado de trabajo deben seguir focalizándose sobre la tipología de contratos. Y si no sería conveniente desviar parte de los esfuerzos y recursos hacia políticas que fomenten la creación de empresas más grandes en sectores con una contratación más estable, como parece ser el sector industrial. Así como a políticas activas que adecuen la formación de la mano de obra más joven a las necesidades del sistema productivo, aunque para ello también habría que mejorar los canales de comunicación e información entre los diferentes agentes intermediadores del mercado de trabajo.

Además, resultaría interesante conocer qué es lo que necesitan las empresas españolas y bajo qué condiciones están dispuestas a contratar a los jóvenes, si con los incentivos introducidos por los cambios legislativos siguen sin fomentar el empleo estable.

\section{Bibliografía.}

Ayllón, S. (2014), "Youth Poverty, Employment, and Leaving the Parental Home in Europe" Review of Income and Wealth 2014, DOI: 10.1111/roiw. 12122

Bentolila, S. y Dolado J.(1994), "Labour flexibility and wages: lessons from Spain”, Economic Policy 18, pp. 53-99

Cahuc P. (2014) "Search, Flows, Job Creations and Destructions", IZA DP No. 81

Cebrián, I., Moreno, G. y Toharia, L. (2011), "La Estabilidad Laboral y los Programas de Fomento de la Contratación Indefinida", Revista de Hacienda Pública, nº 198: pp. (103-130).

Cebrián, I. y Toharia, L. (2008), "La entrada en el mercado de trabajo español: un análisis basado en la Muestra Continua de Vidas Laborales", Revista de economía aplicada, n E-1 (vol.XVI): 137-172. 
Comisión Europea (2007), Flexicurity Pathways. Turning hurdles into stepping stones.

Eurofound (2014), Mapping youth transitions in Europe, Publications Office of the European Union,Luxembourg.

García Pérez, J.I. \& F. Muñoz Bullón (2011), "Transitions into permanent employment in Spain: An empirical analysis for young workers", British Journal of Industrial Relations, vol 49(1), pp. 103-143.

García Pérez, Ioana Marinescu, Judit Vall Castello(2014), "Can FixedTerm Contracts Put Low Skilled Youth on a Better Career Path? Evidence from Spain”. Fedea dt-2014-08.

Gregg, P., Scutella R. and Wadsworth, J. (2004): "Reconciling Workless Measures at the Individual and Household Level: Theory and Evidence from the United States, Britain, Germany, Spain and Australia" CEP Discussion Paper No 635 May 2004

Guamán, Adoración (2013): "De la estabilidad en el empleo a la precariedad laboral por vía de la contratación temporal: la inserción de los jóvenes en el mercado de trabajo como paradigma del trabajo precario", Anuario Facultad de Derecho, Universidad de Alcalá VI(2013) pp.103-136

Güell, M. y Petrongolo, B. (2007), "How Binding Are Legal Limits? Transitions from Temporary to Permanent Work in Spain”, Labour Economics, vol. 14, n 2 , Abril, págs. 153-183.

Hernández Díez, E. y Gentile, A. (2015), "Diseño y aplicación de la garantía juvenil en España". Ponencia en el $V$ Congreso de la Red Española de Política Social.

The Economic Journal (2001), Número especial dedicado al tema "Unemployment scarring", vol. 111, nº. 475, Noviembre.

OCDE (2014), Employment Outlook, 2014.

OIT (2014), Tendencias mundiales del empleo juvenil, 2013. 
Rocha, F. (2012): "El desempleo juvenil en España. Situaciones y recomendaciones políticas". Fundación $1^{\circ}$ de Mayo. Colección Informes $\mathrm{n}^{\mathrm{o}} 50$.

Toharia, L. (dir.) (2005), "El problema de la temporalidad en España: un diagnóstico", Madrid, Ministerio de Trabajo y Asuntos Sociales.

Toharia, L. y Cebrián, I. (2007), La temporalidad en el empleo: atrapamiento y trayectorias, Madrid, Ministerio de Trabajo y Asuntos Sociales. 
160 Cebrián \& Moreno/ Revista de Economía Laboral 12 (2015), 128-163

\section{Anexo}

\section{Cuadro A.1 Modelos de probabilidad de acceso al empleo indefinido}

\begin{tabular}{|c|c|c|c|c|c|c|c|c|c|}
\hline & & & & \multicolumn{6}{|c|}{$\begin{array}{c}\text { Probabilidad de acceder a un contrato indefinido } \\
\text { ordinario }\end{array}$} \\
\hline & & \multicolumn{2}{|c|}{$\begin{array}{l}\text { Probabilidad de } \\
\text { indefinido frente } \\
\text { temporal }\end{array}$} & \multicolumn{2}{|c|}{$\begin{array}{c}\text { Contratos iniciales } \\
\text { bonificados }\end{array}$} & \multicolumn{2}{|c|}{ Conver.bonif } & \multicolumn{2}{|c|}{$\begin{array}{l}\text { Convers.no } \\
\text { bonif. }\end{array}$} \\
\hline & & Prob. & Sig. & Prob. & Sig. & Prob. & Sig. & Prob. & Sig. \\
\hline \multirow{2}{*}{ 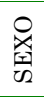 } & Hombre & 1,200 & $* *$ & 0,792 & $* *$ & 0,954 & $* *$ & 0,849 & $* *$ \\
\hline & Mujer (\&) & & & & & & & & \\
\hline \multirow{10}{*}{ 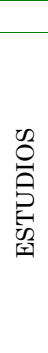 } & EDAD & 1,077 & $* *$ & 0,929 & $* *$ & 0,836 & $* *$ & 1,004 & $* *$ \\
\hline & Sin estud/sin det & & & & & & & & \\
\hline & Est.primarios & 1,065 & $* *$ & 0,894 & $* *$ & 1,539 & $* *$ & 0,747 & $* *$ \\
\hline & FP básica/media & 1,230 & $* *$ & 1,023 & & 5,905 & $* *$ & 1,193 & $* *$ \\
\hline & $\begin{array}{l}\text { Secundarios primer nivel } \\
\text { incompletos }\end{array}$ & 0,947 & $* *$ & 0,779 & $* *$ & 3,292 & $* *$ & 1,122 & $* *$ \\
\hline & Secundarios primer nivel completos & 1,057 & $* *$ & 0,917 & $* *$ & 1,656 & $* *$ & 1,206 & $* *$ \\
\hline & Bachillerato & 0,996 & & 0,981 & & 0,804 & $* *$ & 0,998 & \\
\hline & FP superior & 1,540 & $* *$ & 0,961 & $* *$ & 9,706 & $* *$ & 1,113 & $* *$ \\
\hline & Univ. medios y otros & 1,423 & $* *$ & 1,038 & * & 7,668 & $* *$ & 0,929 & $* *$ \\
\hline & Univ. superiores & 1,938 & $* *$ & 1,053 & $* *$ & 7,776 & $* *$ & 0,767 & $* *$ \\
\hline \multirow{18}{*}{$\sum_{\circlearrowright}^{ঙ}$} & Andalucía (\&) & & & & & & & & \\
\hline & Aragón & 1,976 & $* *$ & 1,179 & $* *$ & 0,788 & $* *$ & 0,907 & $* *$ \\
\hline & Asturias & 1,380 & $* *$ & 1,359 & $* *$ & 1,535 & $* *$ & 0,837 & $* *$ \\
\hline & Baleares & 1,655 & $* *$ & 0,791 & $* *$ & 0,391 & $* *$ & 0,842 & $* *$ \\
\hline & Canarias & 2,107 & $* *$ & 0,800 & $* *$ & 0,350 & $* *$ & 0,913 & $* *$ \\
\hline & Cantabria & 1,437 & $* *$ & 1,149 & $* *$ & 1,319 & $* *$ & 0,903 & $* *$ \\
\hline & Castilla-La Mancha & 1,696 & $* *$ & 0,928 & $* *$ & 0,820 & $* *$ & 0,874 & $* *$ \\
\hline & Castilla y León & 1,714 & $* *$ & 1,120 & $* *$ & 0,892 & $* *$ & 0,672 & $* *$ \\
\hline & Cataluña & 2,980 & $* *$ & 0,860 & $* *$ & 0,355 & $* *$ & 0,617 & $* *$ \\
\hline & C.Valenciana & 1,686 & $* *$ & 0,996 & & 0,824 & $* *$ & 0,888 & $* *$ \\
\hline & Extremadura & 1,245 & $* *$ & 1,494 & $* *$ & 0,713 & $* *$ & 0,603 & $* *$ \\
\hline & Galicia & 1,478 & $* *$ & 1,266 & $* *$ & 1,463 & $* *$ & 0,949 & $* *$ \\
\hline & Madrid & 3,791 & $* *$ & 0,959 & & 0,393 & $* *$ & 0,496 & $* *$ \\
\hline & Murcia & 1,481 & $* *$ & 0,919 & $* *$ & 0,924 & * & 0,996 & \\
\hline & Navarra & 1,663 & $* *$ & 0,899 & $* *$ & 0,948 & & 0,667 & $* *$ \\
\hline & País Vasco & 1,329 & $* *$ & 1,092 & $* *$ & 1,353 & $* *$ & 0,706 & $* *$ \\
\hline & La Rioja & 1,899 & $* *$ & 0,807 & $* *$ & 0,497 & $* *$ & 0,600 & $* *$ \\
\hline & Ceuta y Melilla & 1,863 & $* *$ & 0,521 & $* *$ & 0,366 & $* *$ & 0,645 & $* *$ \\
\hline \multirow{15}{*}{ 严 } & Agricultura y pesca (\&) & & & & & & & & \\
\hline & Ind Extra & 10,104 & $* *$ & 1,135 & & 3,499 & $* *$ & 1,953 & $* *$ \\
\hline & Ind Manuf & 7,062 & $* *$ & 1,474 & $* *$ & 6,914 & $* *$ & 2,315 & $* *$ \\
\hline & Suministro Elect, Agua & 5,093 & $* *$ & 1,345 & $* *$ & 7,954 & $* *$ & 1,689 & $* *$ \\
\hline & Construcc & 3,612 & $* *$ & 1,043 & & 3,182 & $* *$ & 1,716 & $* *$ \\
\hline & Comercio & 8,885 & $* *$ & 1,756 & $* *$ & 3,997 & $* *$ & 1,952 & $* *$ \\
\hline & Hostelería & 6,139 & $* *$ & 1,652 & $* *$ & 0,904 & & 1,254 & $* *$ \\
\hline & Transp y comun & 4,826 & $* *$ & 1,255 & * & 2,176 & $* *$ & 1,460 & $* *$ \\
\hline & Activ Fras y seguros & 6,408 & $* *$ & 1,300 & $* *$ & 7,589 & $* *$ & 1,478 & $* *$ \\
\hline & Inmobil y serv. empresas & 1,791 & $* *$ & 1,864 & $* *$ & 4,241 & $* *$ & 1,605 & $* *$ \\
\hline & Adm. Públ. & 0,571 & $* *$ & 0,333 & $* *$ & 1,597 & $* *$ & 0,857 & $* *$ \\
\hline & Educación & 1,367 & $* *$ & 1,339 & $* *$ & 3,450 & $* *$ & 1,506 & $* *$ \\
\hline & Serv. sanitarios & 2,019 & $* *$ & 1,753 & $* *$ & 3,614 & $* *$ & 2,987 & $* *$ \\
\hline & Hogares & 7,434 & $* *$ & 0,128 & $* *$ & 0,267 & $* *$ & 0,245 & $* *$ \\
\hline & Otras actividades y servicios & 2,068 & $* *$ & 1,751 & $* *$ & 3,070 & $* *$ & 2,279 & $* *$ \\
\hline
\end{tabular}




\begin{tabular}{|c|c|c|c|c|c|c|c|c|c|}
\hline \multirow{9}{*}{$\begin{array}{l}z \\
\text { ○ } \\
\text { U } \\
\text { 岕 } \\
\text { 岁 } \\
0 \\
0\end{array}$} & Fuerzas armadas (\&) & & & & & & & & \\
\hline & Direct y Tecnicos & 5,240 & $* *$ & 0,613 & $* *$ & 0,387 & $* *$ & 1,277 & $* *$ \\
\hline & Administrativos & 5,338 & $* *$ & 1,498 & $* *$ & 0,566 & $* *$ & 1,693 & $* *$ \\
\hline & Serv personales & 4,063 & $* *$ & 1,280 & $* *$ & 0,559 & $* *$ & 1,480 & $* *$ \\
\hline & Dependientes comercio & 3,112 & $* *$ & 1,513 & $* *$ & 0,310 & $* *$ & 1,570 & $* *$ \\
\hline & Agrícolas & 2,326 & $* *$ & 1,378 & $* *$ & 1,087 & & 1,918 & $* *$ \\
\hline & Trabajadores semi-cualificados & 3,688 & $* *$ & 1,626 & $* *$ & 0,682 & $* *$ & 1,655 & $* *$ \\
\hline & Trabajadores no cualificados en servicios & 2,930 & $* *$ & 1,490 & $* *$ & 0,359 & $* *$ & 1,425 & $* *$ \\
\hline & Otros trabajadores no cualificados & 1,841 & $* *$ & 1,917 & $* *$ & 0,499 & $* *$ & 2,623 & ** \\
\hline \multirow{4}{*}{ 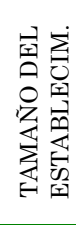 } & Menos de 4 (\&) & & $* *$ & & & & & & \\
\hline & $5-9$ & 0,892 & $* *$ & 0,767 & $* *$ & 0,632 & $* *$ & 1,533 & $* *$ \\
\hline & $10-19$ & 0,795 & $* *$ & 0,640 & $* *$ & 0,647 & $* *$ & 1,646 & $* *$ \\
\hline & 250 o más & 0,363 & $* *$ & 0,526 & $* *$ & 0,902 & * & 1,140 & $* *$ \\
\hline \multirow{2}{*}{$\stackrel{\overbrace{}}{ٍ ٍ ~}$} & Tiempo completo & 1,089 & $* *$ & 0,782 & $* *$ & 0,374 & $* *$ & 0,787 & $* *$ \\
\hline & Tiempo parcial (\&) & & & & & & & & \\
\hline \multirow{2}{*}{\multicolumn{2}{|c|}{$\begin{array}{c}\text { BONIFICACIÓN } \\
\text { VARIACIÓN DEL EMPLEO }\end{array}$}} & 13,508 & $* *$ & & & & & & \\
\hline & & 1,008 & $* *$ & 1,000 & * & 1,006 & * & 0,998 & $* *$ \\
\hline \multirow{25}{*}{ 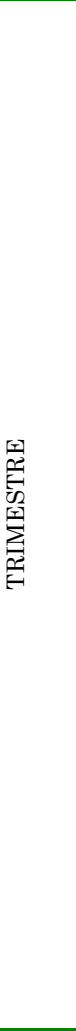 } & $2007 I(\&)$ & & $* *$ & & & & & & \\
\hline & $2007 \mathrm{II}$ & 0,974 & $* *$ & 1,067 & $* *$ & 1,264 & $* *$ & 1,249 & ** \\
\hline & 2007III & 0,972 & $* *$ & 0,932 & $* *$ & 1,192 & $* *$ & 1,242 & $* *$ \\
\hline & 2007IV & 1,065 & $* *$ & 0,947 & $* *$ & 1,201 & $* *$ & 1,208 & $* *$ \\
\hline & $2008 \mathrm{I}$ & 1,235 & $* *$ & 0,861 & $* *$ & 1,184 & $* *$ & 1,288 & $* *$ \\
\hline & $2008 \mathrm{II}$ & 0,987 & $* *$ & 0,911 & $* *$ & 1,268 & $* *$ & 1,388 & $* *$ \\
\hline & $2010 \mathrm{II}$ & 0,592 & $* *$ & 1,004 & & 1,562 & $* *$ & 1,348 & ** \\
\hline & 2010III & 0,579 & $* *$ & 0,025 & $* *$ & 1,077 & $*$ & 0,987 & \\
\hline & 2010IV & 0,703 & $* *$ & 0,019 & $* *$ & 1,004 & & 1,158 & $* *$ \\
\hline & $2011 \mathrm{I}$ & 0,749 & $* *$ & 0,035 & $* *$ & 0,893 & $* *$ & 0,986 & \\
\hline & 2011II & 0,557 & $* *$ & 0,047 & $* *$ & 0,844 & $* *$ & 1,109 & $* *$ \\
\hline & 2011III & 0,493 & $* *$ & 0,026 & $* *$ & 1,084 & * & 1,031 & * \\
\hline & 2011IV & 0,541 & $* *$ & 0,019 & $* *$ & 1,341 & $* *$ & 1,090 & $* *$ \\
\hline & $2012 \mathrm{I}$ & 0,648 & $* *$ & 0,282 & $* *$ & 0,334 & $* *$ & 1,095 & $* *$ \\
\hline & $2012 \mathrm{II}$ & 0,529 & $* *$ & 0,409 & $* *$ & 0,593 & $* *$ & 1,179 & $* *$ \\
\hline & 2012III & 0,491 & $* *$ & 0,223 & $* *$ & 0,559 & $* *$ & 1,117 & $* *$ \\
\hline & 2012IV & 0,560 & $* *$ & 0,187 & $* *$ & 0,560 & $* *$ & 1,105 & $* *$ \\
\hline & $2013 \mathrm{I}$ & 0,635 & $* *$ & 0,220 & $* *$ & 0,629 & $* *$ & 1,224 & $* *$ \\
\hline & 2013II & 0,436 & $* *$ & 0,366 & $* *$ & 0,808 & $* *$ & 1,228 & $* *$ \\
\hline & 2013III & 0,431 & $* *$ & 0,279 & $* *$ & 0,718 & $* *$ & 1,212 & $* *$ \\
\hline & 2013IV & 0,499 & $* *$ & 0,281 & $* *$ & 0,703 & $* *$ & 1,194 & $* *$ \\
\hline & $2014 \mathrm{I}$ & 0,560 & $* *$ & 0,409 & $* *$ & 0,594 & $* *$ & 1,053 & $* *$ \\
\hline & $2014 \mathrm{II}$ & 0,423 & $* *$ & 0,792 & $* *$ & 0,593 & $* *$ & 1,038 & $* *$ \\
\hline & 2014III & 0,431 & $* *$ & 0,697 & $* *$ & 0,608 & $* *$ & 1,044 & $* *$ \\
\hline & 2014IV & 0,517 & $* *$ & 0,770 & $* *$ & 0,575 & $* *$ & 1,075 & $* *$ \\
\hline \multirow{2}{*}{\multicolumn{2}{|c|}{ CONSTANTE }} & 0,001 & $* *$ & & & & & & \\
\hline & & N 40413517 & & N 3067298 & & & & & \\
\hline
\end{tabular}

Fuente: microdatos del registro de contratos SEPE. 2007-14. 
162 Cebrián \& Moreno/ Revista de Economía Laboral 12 (2015), 128-163

\section{Cuadro A.2 Tasa de riesgo resultante del modelo de duración de contratos indefinidos.}

\begin{tabular}{|c|c|c|c|}
\hline & & Tasa de Riesgo & Significatividad \\
\hline \multirow[t]{8}{*}{ Tipo de contrato } & Ordinario TC (\&) & & \\
\hline & Ordinario TP & 1,23 & $* *$ \\
\hline & Inicial bonificado TC & 0,996 & \\
\hline & Inicial bonificado TP & 1,372 & $* *$ \\
\hline & Conversión bonificada TC & 0,996 & $* *$ \\
\hline & Conversión bonificada TP & 0,508 & $* *$ \\
\hline & Conversión no bonificada TC & 0,669 & $* *$ \\
\hline & Conversión no bonificada TP & 0,743 & $* *$ \\
\hline \multicolumn{2}{|l|}{ Mujer } & 1,018 & \\
\hline \multirow{18}{*}{ Comunidad Autónoma } & Andalucía (\&) & & \\
\hline & Aragón & 0,984 & \\
\hline & Asturias & 1,012 & \\
\hline & Baleares & 1,003 & \\
\hline & Canarias & 1,028 & \\
\hline & Cantabria & 1,004 & \\
\hline & Castilla-La Mancha & 0,911 & $* *$ \\
\hline & Castilla-León & 1,034 & \\
\hline & Cataluña & 1,123 & $* *$ \\
\hline & C. Valenciana & 1,149 & $* *$ \\
\hline & Extremadura & 0,968 & \\
\hline & Galicia & 1,046 & \\
\hline & Madrid & 1,21 & $* *$ \\
\hline & Murcia & 0,977 & \\
\hline & Navarra & 1,046 & \\
\hline & País Vasco & 1,011 & \\
\hline & La Rioja & 0,971 & \\
\hline & Ceuta y Melilla & 1,14 & \\
\hline \multirow[t]{2}{*}{ Edad } & $16-24$ & 1,447 & $* *$ \\
\hline & $25-29(\&)$ & & \\
\hline \multirow[t]{16}{*}{ Rama de actividad } & Agricultura & 3,008 & $* *$ \\
\hline & Pesca & 1,613 & $* *$ \\
\hline & Industria Extractiva & 1,186 & \\
\hline & Industria Manufactura (\&) & & \\
\hline & Energía & 0,826 & $* *$ \\
\hline & Construcción & 1,5 & $* *$ \\
\hline & Comercio & 1,319 & $* *$ \\
\hline & Hostelería & 2,007 & $* *$ \\
\hline & Transporte & 1,338 & $* *$ \\
\hline & Intermediarios financieros & 0,98 & \\
\hline & Agencias Inmobiliarias y Serv. Empresas & 1,354 & $* *$ \\
\hline & Administración Pública & 1,597 & $* *$ \\
\hline & Educación & 1,152 & $* *$ \\
\hline & Sanidad & 1,045 & \\
\hline & Otros Servicios y Serv. Personales & 1,389 & $* *$ \\
\hline & Hogares & 0,88 & \\
\hline \multirow[t]{10}{*}{ Grupo de Tarifa (Ocupación) } & No consta $(\&)(+)$ & & \\
\hline & Licenciados & 0,613 & \\
\hline & Ingenieros, Técnicos y Ayudantes & 0,623 & \\
\hline & Jefes Administrativos & 0,586 & \\
\hline & Ayudantes no titulados & 0,589 & \\
\hline & Oficiales Administrativos & 0,652 & $* *$ \\
\hline & Subalternos & 0,759 & $* *$ \\
\hline & Auxiliares Administrativos & 0,809 & $* *$ \\
\hline & Oficiales $1^{\mathrm{a}}$ y $2^{\mathrm{a}}$ & 0,746 & $* *$ \\
\hline & Oficiales $3^{\mathrm{a}}$ y similar & 0,781 & $* *$ \\
\hline
\end{tabular}




\begin{tabular}{|c|c|c|c|}
\hline & Trabajado no cualificado & 0,828 & $* *$ \\
\hline & Menores de 18 & 1,191 & $* *$ \\
\hline Trimestres & $1(\&)$ & & \\
\hline \multirow[t]{3}{*}{2007} & 2 & 1,046 & \\
\hline & 3 & 1,029 & \\
\hline & 4 & 0,979 & \\
\hline Trimestres & 1 & 0,954 & \\
\hline \multirow[t]{3}{*}{2008} & 2 & 0,954 & \\
\hline & 3 & 0,937 & $* *$ \\
\hline & 4 & 0,85 & $* *$ \\
\hline Trimestres & 1 & 0,823 & $* *$ \\
\hline \multirow[t]{3}{*}{2009} & 2 & 0,802 & $* *$ \\
\hline & 3 & 0,785 & $* *$ \\
\hline & 4 & 0,784 & $* *$ \\
\hline Trimestres & 1 & 0,774 & $* *$ \\
\hline \multirow[t]{3}{*}{2010} & 2 & 0,806 & $* *$ \\
\hline & 3 & 0,771 & $* *$ \\
\hline & 4 & 0,802 & $* *$ \\
\hline Trimestres & 1 & 0,748 & $* *$ \\
\hline \multirow[t]{3}{*}{2011} & 2 & 0,773 & $* *$ \\
\hline & 3 & 0,787 & $* *$ \\
\hline & 4 & 0,79 & $* *$ \\
\hline Trimestres & 1 & 0,69 & $* *$ \\
\hline \multirow[t]{3}{*}{2012} & 2 & 0,763 & $* *$ \\
\hline & 3 & 0,787 & $* *$ \\
\hline & 4 & 0,748 & $* *$ \\
\hline Trimestres & 1 & 0,704 & $* *$ \\
\hline \multirow[t]{3}{*}{2013} & 2 & 0,856 & $* *$ \\
\hline & 3 & 0,844 & $* *$ \\
\hline & 4 & 0,818 & $* *$ \\
\hline \multirow[t]{5}{*}{ Tipo de empresa } & Persona Física & 0,959 & $* *$ \\
\hline & Sociedad Anónima (\&) & & \\
\hline & Otras sociedades & 1,029 & $* *$ \\
\hline & Cooperativas y similares & 0,971 & \\
\hline & Sector Público & 0,842 & $* *$ \\
\hline \multirow[t]{9}{*}{ Tamaño del centro de trabajo } & Ningún trabajador & 2,654 & $* *$ \\
\hline & 1 a 4 & 1,314 & $* *$ \\
\hline & 5 a 9 & 1,328 & $* *$ \\
\hline & 10 a 19 & 1,34 & $* *$ \\
\hline & 20 a 49 & 1,212 & $* *$ \\
\hline & 50 a 99 & 1,181 & $* *$ \\
\hline & 100 a 249 & 1,168 & $* *$ \\
\hline & 250 a 499 & 1,283 & $* *$ \\
\hline & 500 y más (\&) & & \\
\hline \multicolumn{2}{|c|}{ Variación del empleo } & 1,004 & \\
\hline \multicolumn{2}{|c|}{ Antigüedad en el sistema de la Seguridad Social } & 1,049 & $* *$ \\
\hline
\end{tabular}

Número de casos en el modelo: 78.712

(\&) Variable de Referencia; ** Significativo al 99 por ciento.

(+)El grupo no consta recoge a jóvenes que están empleados como empleados del hogar.

Fuente: MCVL, 2007-2013. 